\title{
Self-interacting inelastic dark matter in the light of XENON1T excess
}

\author{
Manoranjan Dutta ${ }^{1, *}$ Satyabrata Mahapatra, ${ }^{1, \dagger}$ Debasish Borah, ${ }^{2, \star}$ and Narendra Sahu $\odot^{1, \S}$ \\ ${ }^{1}$ Department of Physics, Indian Institute of Technology Hyderabad, \\ Kandi, Sangareddy 502285, Telangana, India \\ ${ }^{2}$ Department of Physics, Indian Institute of Technology Guwahati, Assam 781039, India
}

(Received 25 January 2021; accepted 27 April 2021; published 19 May 2021)

\begin{abstract}
We propose a self-interacting inelastic dark matter (DM) scenario as a possible origin of the recently reported excess of electron recoil events by the XENON1T experiment. Two quasidegenerate Majorana fermion DM particles interact within themselves via a light hidden sector massive gauge boson and with the standard model particles via gauge kinetic mixing. We also consider an additional long-lived singlet scalar, which helps in realizing correct dark matter relic abundance via a hybrid setup comprising both freeze-in and freeze-out mechanisms. While being consistent with the required DM phenomenology along with sufficient self-interactions to address the small-scale issues of cold dark matter, the model with GeV-scale DM can explain the XENON1T excess via inelastic down-scattering of the heavier DM component into the lighter one. All these requirements leave a very tiny parameter space, keeping the model very predictive for near-future experiments.
\end{abstract}

DOI: 10.1103/PhysRevD.103.095018

\section{INTRODUCTION}

There exists a convincing amount of evidence suggesting the presence of a nonluminous, nonbaryonic form of matter in the present Universe, popularly known as dark matter (DM). This form of matter constitutes a significant portion of galaxies, clusters, and the entire Universe. Data from cosmology experiments like Planck, which measures the cosmic microwave background (CMB) anisotropies very precisely, predict the amount of DM in the present Universe to be around $26.8 \%$ of the present Universe's energy density. In terms of density parameter $\Omega_{\mathrm{DM}}$ and $h=$ Hubble parameter $/\left(100 \mathrm{~km} \mathrm{~s}^{-1} \mathrm{Mpc}^{-1}\right)$, the present $\mathrm{DM}$ abundance is conventionally reported as [1] $\Omega_{\mathrm{DM}} h^{2}=$ $0.120 \pm 0.001$ at $68 \% \mathrm{C}, \mathrm{L}$. Similar evidence exists in galactic and cluster scales as well, collected over a long period of time since the 1930s [2-4]. It should be noted that the Planck estimate of present DM abundance relies upon the standard model of cosmology or $\Lambda \mathrm{CDM}$ cosmology, which has been very successful in the overall description of our Universe at large scale [greater than or equal to $\mathcal{O}(\mathrm{Mpc})]$. Here, CDM refers to cold dark matter, while

\footnotetext{
ph18resch11007@iith.ac.in

ph18resch11001@iith.ac.in

*dborah@iitg.ac.in

${ }^{\S}$ nsahu@phy.iith.ac.in
}

Published by the American Physical Society under the terms of the Creative Commons Attribution 4.0 International license. Further distribution of this work must maintain attribution to the author(s) and the published article's title, journal citation, and DOI. Funded by SCOAP.
$\Lambda$ denotes the cosmological constant or dark energy. CDM, a pressureless or collisionless fluid, acts like a seed for structure formation providing the required gravitational potential well for ordinary matter to collapse and form structures. Since none of the standard model (SM) particles can be a viable CDM candidate, several beyond standard model proposals have been put forward, out of which the weakly interacting massive particle (WIMP) paradigm is the most widely studied one. In this framework, a WIMP candidate typically having interactions and mass in the electroweak regime naturally satisfies the correct DM relic abundance, a remarkable coincidence often referred to as the WIMP miracle [5].

While $\Lambda \mathrm{CDM}$ is in excellent agreement with large-scale structure of the Universe, there exist some discrepancies between its prediction and observations, particularly at small scales. In particular, the too-big-to-fail, missing satellite, and core-cusp problems are three such well-known cases where $\Lambda \mathrm{CDM}$ appears to be in conflict with observations. For recent reviews of these issues and possible solutions, please see Refs. [6,7]. One interesting solution to this puzzle was proposed by Spergel and Steinhardt [8], who considered an alternative to collisionless CDM in terms of self-interacting dark matter (SIDM). ${ }^{1}$ While SIDM solves the problems at small scales, it reproduces the CDM halos at large radii, thus consistent with observations. This is simply due to the fact that the self-interacting scattering rate is proportional to DM density. The required selfinteraction rate is often quantified as a ratio of the cross

\footnotetext{
${ }^{1}$ See Ref. [9] for earlier studies.
} 
section to DM mass as $\sigma / m \sim 1 \mathrm{~cm}^{2} / \mathrm{g} \approx 2 \times 10^{-24} \mathrm{~cm}^{2} / \mathrm{GeV}$ [10-15]. Such self-interacting cross sections can be naturally realized in models with very light mediator. For such a scenario, self-interactions can be shown to be stronger for smaller DM velocities such that the DM can have large impact on small-scale structures while being consistent with usual CDM predictions at larger scales [10-13,16-19]. From a particle physics point of view, such self-interactions can be naturally realized in Abelian gauge extensions of the SM. The DM sector cannot be completely hidden and there should be some coupling of the mediator with SM particles as well, which can ensure that DM and SM sectors were in thermal equilibrium in the early Universe. The same coupling can also be probed at DM direct detection experiments as well $[20,21]$. Several model-building efforts have been made to realize such scenarios. For example, see Refs. [22-27] and references therein.

DM with light mediators has also received attention very recently after the XENON1T collaboration published its latest results in June 2020 in which it reports the observation of an excess of electron recoil events over the background in the recoil energy $E_{r}$ in a range $1-7 \mathrm{keV}$, peaked around $2.4 \mathrm{keV}$ [28]. While the excess can be explained by solar axions at $3.5 \sigma$ significance or neutrinos with magnetic moment at $3.2 \sigma$ significance, both these interpretations face stringent stellar cooling bounds. While there is also room for possible tritium backgrounds in the detector, which the XENON1T Collaboration neither confirms nor rules out at this stage, there have been several interesting new physics proposals in the literature. For example, see Refs. [29-47] and references therein. The DM interpretations out of these examples typically have a light mediator via which DM interacts with electrons. The recoil can occur due to either light boosted DM or inelastic up- or down-scattering [35-44,48-54].

Thus, we noticed that in a class of models the DM interpretation of the XENON1T excess and SIDM phenomenology rely on light mediators. This motivates us to propose a common platform to show that the self-interaction of DM arising via light mediators in such models can also give rise the observed XENON1Texcess. In other words, the proposed scenario provides a unique way of probing the parameter space of SIDM at direct DM search experiments like XENON1T. To be more specific, we consider a dark sector consisting of sub-GeV inelastic DM with $\mathrm{keV}$-scale mass splitting and a corresponding massive vector boson $Z^{\prime}$ $[35,40]$. Unlike earlier works where $\mathrm{DM}$ and $Z^{\prime}$ masses are in the same regime so that DM relic is governed by resonant $2 \rightarrow 2$ annihilations, here we consider light mediators (order of magnitude lighter than DM mass) motivated from the SIDM point of view. While the self-interaction of DM is realized via $Z^{\prime}$ exchange, the latter can mix with $U(1)_{Y}$ gauge boson to provide a unique portal for detecting the DM at direct search experiments. The scalar field which leads to spontaneous breaking of dark sector gauge symmetry also induces a tiny Majorana mass to a singlet Dirac fermion field, leading to an inelastic DM scenario $[55,56]$. In this setup, we first find the DM parameter space consistent with velocity-dependent self-interaction rates explaining the data at the scale of clusters, galaxies, and dwarf galaxies. We then confront the SIDM parameter space with the observed XENON1T electron excess while being consistent with other experimental bounds. We show that these two requirements make pure thermal relic DM insufficient to produce the observed relic, and therefore we consider a hybrid setup where both freeze-out and freeze-in mechanisms can play nontrivial roles in generating the DM relic. As we discuss in the upcoming sections, a long-lived scalar singlet has to be invoked whose late decay into DM helps in generating correct DM relic in such a hybrid setup.

This paper is organized as follows. In Sec. II, we briefly discuss our model, and this is followed by the analysis for dark matter self-interaction in Sec. III. In Sec. IV, we discuss production of self-interacting DM from a hybrid of freeze-in and freeze-out formalism. In Sec. V, we discuss the possible origin of XENON1T excess in our model. We finally summarize our results and conclude in Sec. VI.

\section{MODEL}

We consider a simple Abelian extension of the SM. Under this $U(1)_{X}$ gauge symmetry, the SM fields do not have any charge, while there exists a SM singlet Dirac fermion $\Psi$ with $U(1)_{X}$ charge 1 . An additional scalar $\Phi$, singlet under the SM gauge symmetry, having $U(1)_{X}$ charge -2 is introduced which not only breaks the new gauge symmetry spontaneously but also splits the Dirac fermion into two pseudo-Dirac components as we discuss below. The Dirac fermion $\Psi$ is identified as the DM field. The relevant part of the DM Lagrangian is

$$
\begin{aligned}
\mathcal{L}_{\mathrm{DM}}= & i \bar{\Psi} \gamma^{\mu} D_{\mu} \Psi-M\left(\bar{\Psi}_{L} \Psi_{R}+\bar{\Psi}_{R} \Psi_{L}\right)-\left(y_{L} \Phi \overline{\left(\Psi_{L}\right)^{c}} \Psi_{L}\right) \\
& +\left(y_{R} \Phi \overline{\left(\Psi_{R}\right)^{c}} \Psi_{R}+\text { H.c. }\right)+\frac{\epsilon}{2} B^{\alpha \beta} Y_{\alpha \beta},
\end{aligned}
$$

where $D_{\mu}=\partial_{\mu}+i g^{\prime} Z_{\mu}^{\prime}$ and $B^{\alpha \beta}, Y_{\alpha \beta}$ are the field strength tensors of $U(1)_{X}$ and $U(1)_{Y}$, respectively, and $\epsilon$ is the kinetic mixing between them. Lagrangian involving the singlet scalar can be written as

$$
\begin{aligned}
\mathcal{L}_{\Phi}= & \left(D_{\mu} \Phi\right)^{\dagger}\left(D^{\mu} \Phi\right)+m_{\Phi}^{2} \Phi^{\dagger} \Phi-\lambda_{\phi}\left(\Phi^{\dagger} \Phi\right)^{2} \\
& -\lambda_{\Phi H}\left(\Phi^{\dagger} \Phi\right)\left(H^{\dagger} H\right),
\end{aligned}
$$

where $H$ is the SM Higgs doublet. The scalar fields which acquire a nonzero vacuum expectation value (VEV) can be represented as

$$
H=\left(\begin{array}{c}
h^{+} \\
\frac{\left(h+v+i h^{I}\right)}{\sqrt{2}}
\end{array}\right), \quad \Phi=\frac{\phi+u+i \phi^{I}}{\sqrt{2}} .
$$


The singlet scalar VEV gives rise to $U(1)_{X}$ gauge boson mass $M_{Z^{\prime}}=2 g^{\prime} u$, while Higgs doublet gives rise to the usual SM particle masses.

The scalar singlet $\Phi$ also breaks $U(1)_{X}$ spontaneously down to a remnant $Z_{2}$ symmetry under which $\Psi_{L, R}$ are odd while all other fields are even. As a result, $\Psi_{L}$ and $\Psi_{R}$ combine to give a stable DM candidate in the low-energy effective theory. The VEV of $\Phi$ also generates Majorana masses for fermion DM: $m_{L}=y_{L} u / \sqrt{2}$ and $m_{R}=y_{R} u / \sqrt{2}$ for $\Psi_{L}$ and $\Psi_{R}$, respectively. We assume $m_{L}, m_{R} \ll M$. As a result, the Dirac fermion $\Psi=\Psi_{L}+\Psi_{R}$ splits into two pseudo-Dirac states $\psi_{1}$ and $\psi_{2}$ with masses $M_{1}=M-m_{+}$ and $M_{2}=M+m_{+}$, where $m_{ \pm}=\left(m_{L} \pm m_{R}\right) / 2$. The DM Lagrangian after spontaneous symmetry breaking can be written as

$$
\begin{aligned}
\mathcal{L}_{\mathrm{DM}}= & \frac{1}{2} \overline{\psi_{1}} \gamma^{\mu} \partial_{\mu} \psi_{1}+\frac{1}{2} \overline{\psi_{2}} \gamma^{\mu} \partial_{\mu} \psi_{2}-\frac{1}{2} M_{1} \overline{\psi_{1}} \psi_{1}-\frac{1}{2} M_{2} \overline{\psi_{2}} \psi_{2}+\frac{\epsilon}{2} B^{\alpha \beta} Y_{\alpha \beta} \\
& +i g^{\prime} Z_{\mu}^{\prime} \overline{\psi_{1}} \gamma^{\mu} \psi_{2}+\frac{1}{2} g^{\prime} Z_{\mu}^{\prime} \frac{m_{-}}{M}\left(\overline{\psi_{2}} \gamma^{\mu} \gamma^{5} \psi_{2}-\overline{\psi_{1}} \gamma^{\mu} \gamma^{5} \psi_{1}\right) \\
& +\frac{1}{2}\left(y_{L} \cos ^{2} \theta-y_{R} \sin ^{2} \theta\right) \overline{\psi_{1}} \psi_{1} \phi+\frac{1}{2}\left(y_{R} \cos ^{2} \theta-y_{L} \sin ^{2} \theta\right) \overline{\psi_{2}} \psi_{2} \phi
\end{aligned}
$$

where $\sin \theta \approx m_{-} / M$. The mass splitting between the two mass eigenstates is given by $\Delta m=M_{2}-M_{1}=2 m_{+}=$ $\left(y_{L}+y_{R}\right) \frac{u}{\sqrt{2}}$. To address the XENON1T anomaly, we take $\Delta m \sim 2 \mathrm{keV}$. While we stick to such minimal DM models in this work, such Abelian gauge extensions can be motivated from other phenomena like the origin of light neutrino masses as well, as discussed in several works including Refs. [40,57-67].

\section{DARK MATTER SELF-INTERACTION}

As mentioned before, we have an inelastic DM scenario where two DM components with tiny mass splitting of $\mathrm{keV}$ scale can populate the Universe. We are also considering a light mediator of DM interactions, which is motivated from the SIDM solution to structure formation problems. See Refs. [68-70] for earlier studies on self-interacting inelastic DM which were primarily motivated from the requirements of avoiding strong direct detection constraints or to explain some anomalous observations at indirect detection experiments like monochromatic photon lines. While in our model DM candidates can interact among themselves either via scalar or vector mediators, we consider only the vector mediator or $Z^{\prime}$ to be light and hence consider this only to constrain the parameter space from required self-interactions. The relevant Lagrangian for DM interactions with $Z^{\prime}$ can be rewritten as

$\mathcal{L}=i g^{\prime} Z_{\mu}^{\prime} \overline{\psi_{1}} \gamma^{\mu} \psi_{2}+\frac{1}{2} g^{\prime} Z_{\mu}^{\prime} \frac{m_{-}}{M}\left(\overline{\psi_{2}} \gamma^{\mu} \gamma^{5} \psi_{2}-\overline{\psi_{1}} \gamma^{\mu} \gamma^{5} \psi_{1}\right)$.

Ignoring the second term which is suppressed by $m_{-} / M$, we can write down the corresponding potential for two Majorana fermion DM particles with a light mediator of dark photon type as [68-71]

$$
V(r)=\left(\begin{array}{cc}
0 & -\alpha e^{M_{Z}^{\prime} r} \\
-\alpha e^{M_{Z}^{\prime} r} & 2 \Delta m
\end{array}\right) .
$$

The two-body Schrodinger equation for relative motion is

$$
\frac{1}{M_{\psi}} \nabla^{2} \Psi(\vec{r})=\left(V(r)-M_{\psi} v^{2}\right) \Psi(\vec{r}),
$$

where $M_{\psi}$ is the mass of the dark matter, ignoring the tiny mass splitting $\Delta m ; \mathrm{v}$ is the individual velocity of either of the dark matter particles in the center-of-mass frame (half the relative velocity); $\Delta m$ is the mass splitting between two DM candidates; and $\Psi(\vec{r})$ is the wave function. Defining dimensionless parameters, $\epsilon_{v}=\frac{v}{\alpha}, \epsilon_{\delta}=\sqrt{\frac{2 \Delta m}{M_{\psi} \alpha^{2}}}$, and $\epsilon_{\phi}=\frac{M_{Z^{\prime}}}{M_{\psi} \alpha}$ and writing $r \Psi(\vec{r})=\psi(r)$, the s-wave Schrodinger equation is given by

$$
\psi^{\prime \prime}(r)=\left(\begin{array}{cc}
-\epsilon_{v}^{2} & -\frac{e^{e} Z^{r}}{r} \\
-\frac{e^{e} Z^{r}}{r} & \epsilon_{\delta}^{2}-\epsilon_{v}^{2}
\end{array}\right) \psi(r) .
$$

As shown in Feynman diagrams of Fig. 1, the DM candidates of one type can scatter off each other while remaining in the same state, only at one-loop level, due to the off-diagonal nature of DM-mediator couplings. Using these, we constrain the DM parameter space from the required self-interactions at different scales while considering the mass splitting between the two DM
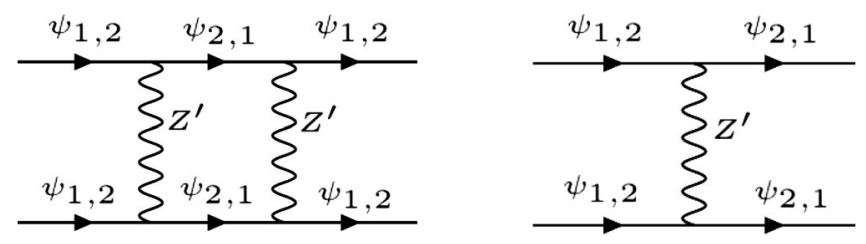

FIG. 1. Feynman diagrams for self-interaction. 
candidates to be $2 \mathrm{keV}$, as favored from XENON1T excess. The relevant cross sections are given in the Appendix A 1. For a more general analysis, one may refer to Ref. [68].

Using these self-interaction cross sections and using the required $\sigma / \mathrm{m}$ from astrophysical observations at different scales, we constrain the parameter space of the model in terms of DM $\left(\psi_{1,2}\right)$ and mediator $Z^{\prime}$ masses. As our study is motivated from explaining the XENON1T excess, we keep the required mass splitting between two DM candidates to be $2 \mathrm{keV}$. In Fig. 2, we show the allowed parameter space in DM mass versus the $Z^{\prime}$ mass plane, which gives rise to the required DM self-interaction cross section $(\sigma / m)$ in the range $0.1-1 \mathrm{~cm}^{2} / \mathrm{g}$ for clusters $(v \sim 1000 \mathrm{~km} / \mathrm{s})$. The corresponding regions of parameter space for galaxies $(v \sim 200 \mathrm{~km} / \mathrm{s})$ and dwarf galaxies $(v \sim 10 \mathrm{~km} / \mathrm{s})$ are shown in Figs. 3 and 4, respectively. It should be noted that for dwarf galaxies, due to smaller DM velocities, we do not get a sufficient self-interaction cross section $(\sigma / \mathrm{m})$ from upscattering processes in the entire parameter space considered, and hence the corresponding plot is not shown in Fig. 4. This is due to the fact that lighter DM, due to low velocities, does not have sufficient kinetic energy to scatter efficiently into heavier DM, resulting in a large self-interaction cross section. We will finally compare these regions of parameter space of GeV-scale DM mass in the context of XENON1T excess and other phenomenological constraints.

The self-interaction cross section per unit mass of DM as a function of average collision velocity is shown in Fig. 5 as measured from astrophysical data. The data include measurements from dwarfs (red), Low surface brightness (LSB)
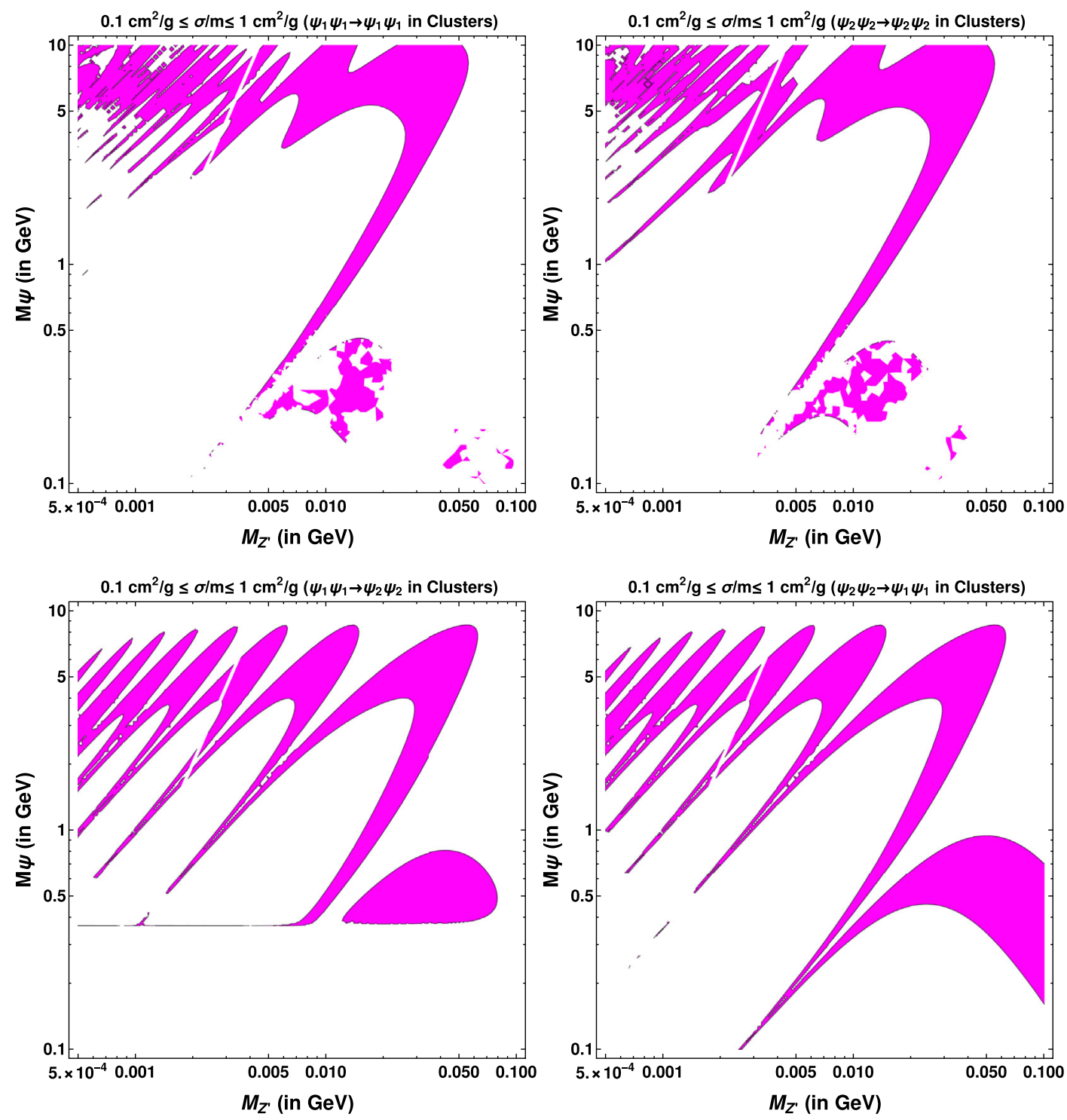

FIG. 2. Self-interaction cross section $(\sigma / \mathrm{m})$ in the range $0.1-1 \mathrm{~cm}^{2} / \mathrm{g}$ (light pink colored region) for clusters $(v \sim 1000 \mathrm{~km} / \mathrm{s})$. Top left (right) panel: elastic scattering of ground (excited) to ground (excited) state. Bottom left (right) panel: up- (down-)scattering of ground (excited) to excited (ground) state. 

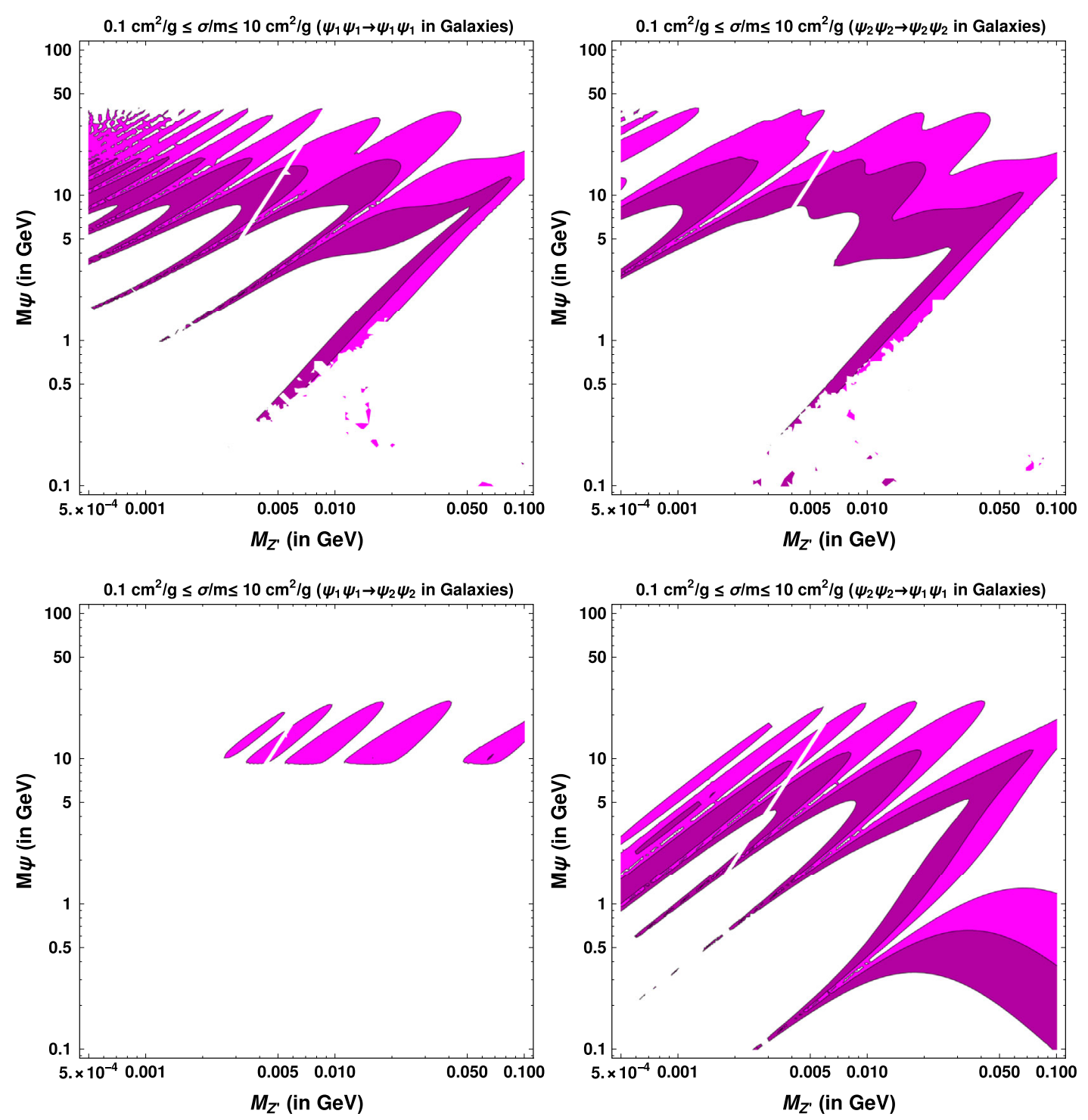

FIG. 3. Self-interaction cross section $(\sigma / m)$ in the range $0.1-10 \mathrm{~cm}^{2} / \mathrm{g}$ for galaxies $(v \sim 200 \mathrm{~km} / \mathrm{s})$. Light pink colored region represents the parameter space where $0.1 \mathrm{~cm}^{2} / \mathrm{g}<\sigma / m<1 \mathrm{~cm}^{2} / \mathrm{g}$, dark pink color represents regions of parameter space where $1 \mathrm{~cm}^{2} / \mathrm{g}<\sigma / m<10 \mathrm{~cm}^{2} / \mathrm{g}$. Top left (right) panel: elastic scattering of ground (excited) to ground (excited) state. Bottom left (right) panel: up- (down-)scattering of ground (excited) to excited (ground) state.

Galaxies (blue), and clusters (green) [17,72]. The purple dashed curve corresponds to the velocity-dependent cross section from our model for a particular set of benchmark values (i.e., $M_{\psi}=1 \mathrm{GeV}, M_{Z^{\prime}}=50 \mathrm{MeV}$, and $\alpha=0.001$ ) allowed from all relevant phenomenological constraints. It is clear from the figure that the model proposed here can explain the astrophysical observation of self-interaction of DM appreciably well. See Ref. [73] for discussions on astrophysical probes of such inelastic DM with a light mediator.

\section{DARK MATTER PRODUCTION}

While several production regimes for self-interacting DM exist in the literature, we first consider the usual $2 \leftrightarrow 2$ vector portal interactions. While DM can interact with itself via $Z^{\prime}$ as well as singlet scalar interactions, we consider the vector portal to be dominant due to light $Z^{\prime}$. On the other hand, DM can interact with the SM bath only via kinetic mixing of neutral vector bosons. These dominant number changing processes are shown in Fig. 6. While DM-SM interactions via kinetic mixing is responsible for production of DM from the thermal bath, the dark sector interactions can be important to decide the final abundance of DM. Since from the SIDM point of view we consider heavier DM mass compared to the mediator $m_{\psi_{1,2}}>m_{Z^{\prime}}$, DM can have a large annihilation cross section to $Z^{\prime}$ affecting its relic abundance. For example, the thermal averaged cross section for the t-channel process $\psi_{1} \psi_{1} \rightarrow Z^{\prime} Z^{\prime}$ shown in the left panel of Fig. 6 is 

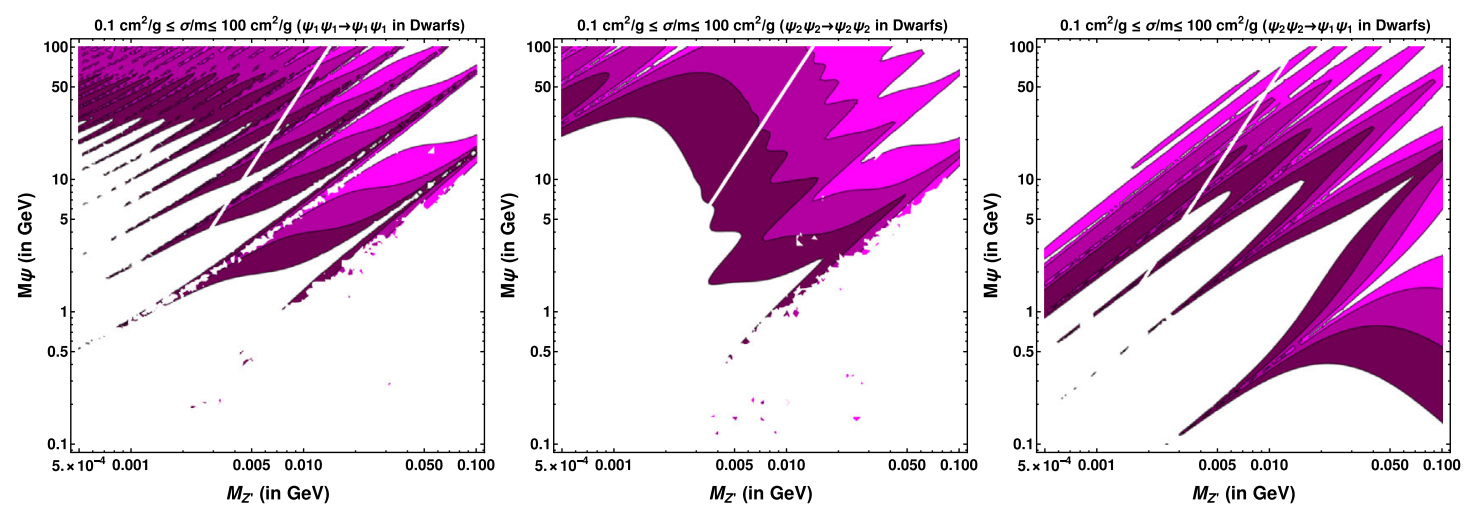

FIG. 4. Self-interaction cross section in the range $0.1-100 \mathrm{~cm}^{2} / \mathrm{g}$ for dwarfs $(v \sim 10 \mathrm{~km} / \mathrm{s})$. Light pink color represents regions of parameter space where $0.1 \mathrm{~cm}^{2} / \mathrm{g}<\sigma / m<1 \mathrm{~cm}^{2} / \mathrm{g}$; dark pink color represents regions of parameter space where $1 \mathrm{~cm}^{2} / \mathrm{g}<\sigma / m<10 \mathrm{~cm}^{2} / \mathrm{g}$; maroon color represents regions of parameter space where $10 \mathrm{~cm}^{2} / \mathrm{g}<\sigma / m<100 \mathrm{~cm}^{2} / \mathrm{g}$. Left (middle) panel: elastic scattering of ground (excited) to ground (excited) state. Right panel: down-scattering of excited to ground state.

$$
\langle\sigma v\rangle \sim \frac{\pi \alpha_{x}^{2}}{M_{\psi}^{2}},
$$

where $\alpha_{x}=g^{\prime 2} /(4 \pi)$ and for typical gauge coupling and DM mass of our interest we have $\alpha_{x} \sim 0.001, M_{\psi} \sim 1 \mathrm{GeV}$. This leads to a cross section which is at least 2 orders of magnitude larger compared to the typical annihilation cross section of thermal DM. This reduces the relic abundance by same orders of magnitude, as seen from Fig. 7 showing the comoving number density of DM, assuming it to be a purely thermal relic. Before calculating DM relic, we first compare rates of different annihilation processes. Note that for the

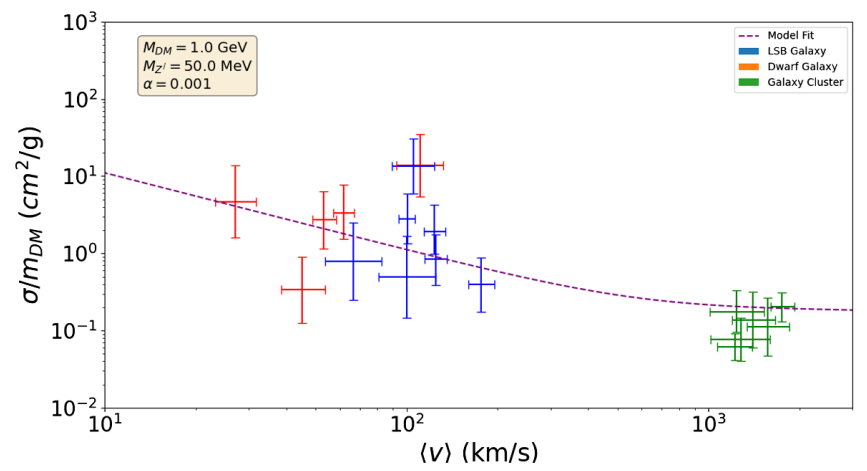

FIG. 5. The self-interaction cross section per unit mass of DM as a function of average collision velocity.

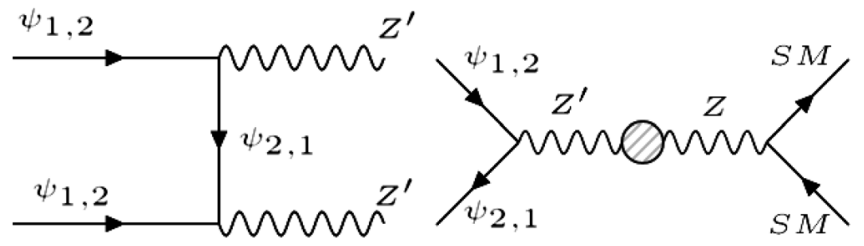

FIG. 6. Feynman diagrams for dominant number changing processes of DM. purpose of numerical analysis the model has been implemented in LanHEP [74] and CALCHEP [75], and the cross sections required has been fed into Mathematica [76] from CALCHEP.

Although dark sector interaction rates are large as mentioned above, the DM-SM interactions are suppressed due to tiny kinetic mixing chosen to realize the required XENON1T excess. We check the relevant DM-SM processes and find that for the chosen sub-GeV regime and kinetic mixing DM never attains chemical equilibrium with the SM bath. While relevant cross sections are given in Appendix A 2, we compare different interaction rates in Fig. 8. In calculating the interaction rates, we consider the light SM degrees of freedom to be in equilibrium, while the

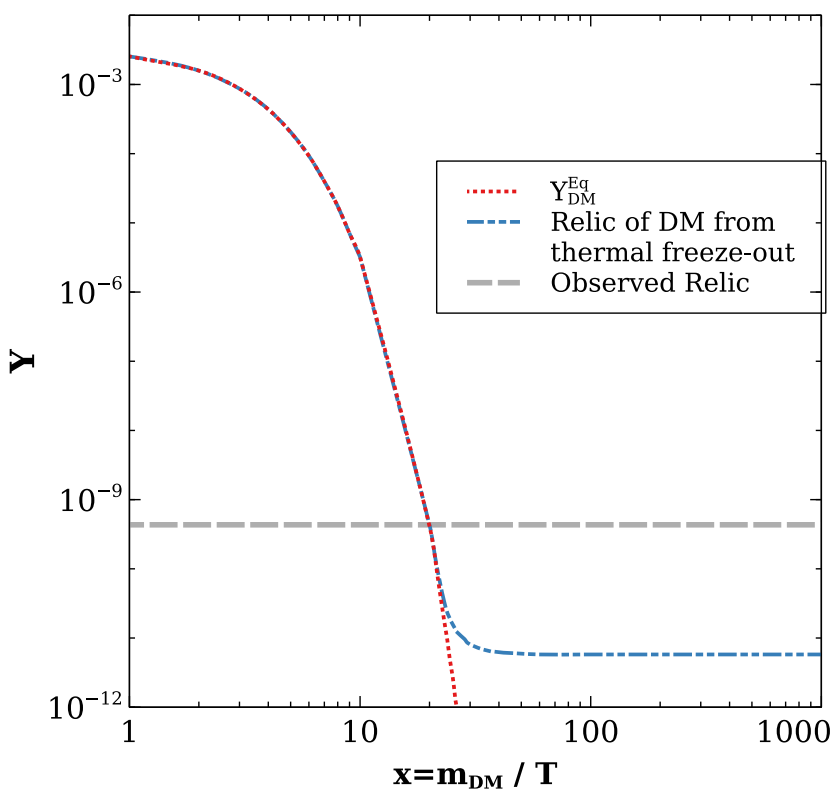

FIG. 7. Relic abundance of DM assuming it to be produced thermally in the early Universe followed by thermal freeze-out. The thermal relic is underabundant by 2 orders of magnitudes. 


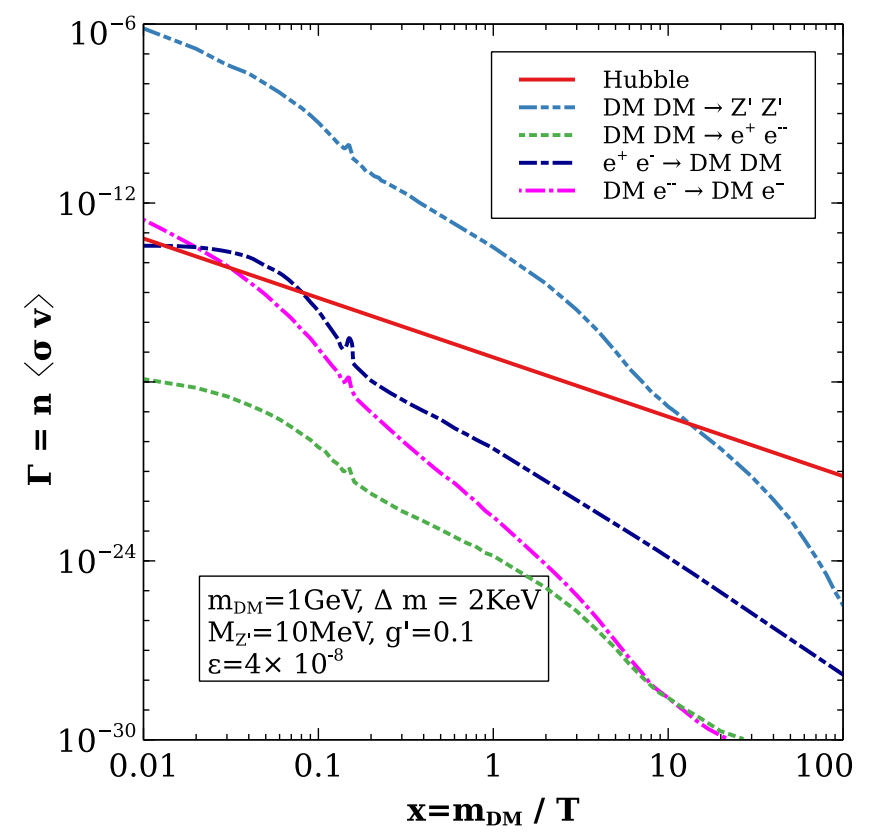

FIG. 8. Decoupling of different processes from the thermal plasma.

DM number density is calculated by solving the appropriate Boltzmann equation considering freeze-in production of DM [77] from the SM bath. This happens dominantly from $2 \rightarrow 2$ processes where SM fermions in equilibrium at $\mathrm{GeV}$ temperatures can contribute to the production of DM. Since the production happens from the thermal bath, it saturates at a temperature similar to that of DM mass. On the other hand, DM produced this way keeps annihilating into $Z^{\prime}$ bosons due to large self-interactions further diluting the DM abundance. Clearly, almost all the $2 \rightarrow 2$ processes remain out of equilibrium as the corresponding interaction rates remain below Hubble expansion rate seen from Fig. 8. Only the DM annihilation rate into the $Z^{\prime}$ boson remains in equilibrium for a longer epoch, while DM-SM kinetic equilibrium is reached for a very short epoch. We also check that the freeze-in production of DM from thermal bath, followed by dark sector freeze-out, is insufficient to produce the correct DM relic for the region of our interest. This is due to the large annihilation rates of DM into $Z^{\prime}$ bosons keeping DM underabundant after dark sector freeze-out. This requires an additional source of DM relic which we consider to be a SM singlet scalar $\eta$. The singlet scalar freezes out in the early Universe via the process $\eta^{\dagger} \eta \rightarrow H^{\dagger} H$ and decays back to DM after the dark sector freezes out, thus filling the deficit in relic abundance. The relevant Lagrangian for $\eta$ decay is given by

$$
\mathcal{L}=\frac{1}{2} \lambda_{1} \overline{\psi_{1}} \psi_{1} \eta+\frac{1}{2} \lambda_{2} \overline{\psi_{2}} \psi_{2} \eta
$$

If the thermal averaged annihilation cross section of $\eta^{\dagger} \eta \rightarrow$ $H^{\dagger} H$ is smaller than the usual freeze-out cross section of a
WIMP, i.e., $\langle\sigma|v|\rangle_{F}=3 \times 10^{-26} \mathrm{~cm}^{3} / s$, then the relic of $\eta$ can be larger than the observed DM abundance. As a result, the late decay of $\eta \rightarrow \psi_{i} \psi_{j}$ can give rise to ample amount of DM. In Eq. (12), we use appropriate Boltzmann equations to get the correct relic density of DM. While we incorporate this additional scalar singlet $\eta$ only to satisfy DM relic through its late decay, it can serve other purposes as well. One such possibility is to realize cosmic inflation. There have been proposals where a single scalar field is shown to play the role of inflation as well as thermal DM whose relic is generated via usual freeze-out. For example, see Ref. [78] and references therein. The same idea can be implemented here as well while noting that the scalar field is not perfectly stable but decays at late epochs into the DM. We, however, do not discuss such additional roles the scalar singlet might play in our minimal scenario discussed here.

From Fig. 8, it is evident that the process $D M e \rightarrow D M e$, which is responsible for keeping both the dark and visible sectors in kinetic equilibrium, decouples around $x \sim 0.03$, after which the temperature of the dark sector (denoted by $T^{\prime}$ ) evolves independently of the thermal bath (temperature $T)$ until $x \sim 100$ when all the dark sector particles becomes nonrelativistic (and hence cease to contribute to the relativistic degrees of freedom). Between these two epochs, the ratio of the two temperatures can be obtained by conserving the total entropy separately in the two sectors. Considering the kinetic decoupling temperature to be $T_{D}$, we can relate the temperature of the two sectors as

$$
\frac{T^{\prime}}{T}=\left(\frac{g_{* s}^{\mathrm{SM}}(T)}{g_{* S}^{\mathrm{SM}}\left(T_{D}\right)}\right)^{1 / 3}
$$

Here, $g_{* s}^{\mathrm{SM}}(T)$ is the relativistic entropy degrees of freedom in the standard model, which goes into the calculation of relativistic entropy density $s(T)=\frac{2 \pi^{2}}{45} g_{* S}(T) T^{3}$. Since the above relation (10) is for $T<T_{D}$, we naturally have $g_{* s}^{\mathrm{SM}}(T)<g_{* s}^{\mathrm{SM}}\left(T_{D}\right)$ leading to $T^{\prime}<T$. This is also understood from the fact that the SM bath temperature receives additional entropy contributions from the species which keep getting decoupled gradually. Within the decoupled dark sector itself, the DM particles can transfer their entropies into lighter $Z^{\prime}$ bosons once $T^{\prime}$ falls below DM mass. This corresponds to an enhancement of dark sector temperature for $T^{\prime}<m_{\mathrm{DM}}$ by $(13 / 6)^{1 / 3}$, a factor close to unity. We have ignored this additional enhancement in the calculations.

Because of the different temperatures of dark sector and SM bath after some epoch, we accordingly divide the range of integration for solving the Boltzmann equations as follows:

(i) From the epoch of reaching kinetic equilibrium between DM-SM sectors until $x<0.03$ (see Fig. 8), both the dark and the visible sectors share the same temperature $T=T^{\prime}$. 
(ii) One with $0.03<x<100$ where the dark sector is decoupled from the thermal bath and its temperature evolves according to (10).

Accordingly, one can define a new dimensionless parameter and relate to the usual parameter $x=\frac{m_{\mathrm{DM}}}{T}$ as

$$
x^{\prime}=\frac{m_{\mathrm{DM}}}{T^{\prime}}=\left(\frac{T}{T^{\prime}}\right) x
$$

We can now write down the Boltzmann equations for two DM candidates $\psi_{1,2}$ and the scalar singlet $\eta$ whose late decays into DM are crucial to generate correct DM relic. Unlike DM whose interactions with the SM bath are suppressed due to tiny kinetic mixing, the scalar singlet can be in thermal equilibrium with the SM due to large quartic couplings followed by freeze-out. ${ }^{2}$ Thus, we define comoving number densities of these particles as $Y_{\psi_{1.2}}=$ $n_{\psi_{1.2}} / s^{\prime}\left(T^{\prime}(T)\right), Y_{\eta}=n_{\eta} / s(T)$. The relevant coupled Boltzmann equations can then be written as

$$
\begin{aligned}
\frac{d Y_{\eta}}{d x^{\prime}}= & -\frac{s\left(M_{\psi}\right)}{x^{\prime 2} H\left(M_{\psi}\right)\left(\frac{T^{\prime}}{T}\right)}\langle\sigma v\rangle_{\eta \eta \rightarrow H H}\left(Y_{\eta}^{2}-\left(Y_{\eta}^{e q}\right)^{2}\right)-\frac{x^{\prime}\left(\frac{T^{\prime}}{T}\right)^{2}\left(\left\langle\Gamma_{\eta \rightarrow \overline{\psi_{1}} \psi_{1}}\right\rangle+\left\langle\Gamma_{\eta \rightarrow \overline{\psi_{2}} \psi_{2}}\right\rangle\right)}{H\left(M_{\psi}\right)} Y_{\eta} ; \\
\frac{d Y_{\psi_{1}}}{d x^{\prime}}= & \left(\frac{T^{\prime}}{T}\right)^{2}\left[\frac { s ( M _ { \psi } ) } { x ^ { \prime 2 } H ( M _ { \psi } ) } ( \frac { g _ { * s } ^ { \prime } ( T _ { D } ) } { g _ { * s } ( T _ { D } ) } ) \left(\langle\sigma v\rangle_{e^{+} e^{-} \rightarrow \psi_{1} \psi_{1}}\left(Y_{\psi_{1}}^{e q}\right)^{2}-\langle\sigma v\rangle_{\psi_{1} \psi_{1} \rightarrow Z^{\prime} Z^{\prime}} Y_{\psi_{1}}^{2}\right.\right. \\
& \left.\left.+\langle\sigma v\rangle_{\psi_{2} \psi_{2} \rightarrow \psi_{1} \psi_{1}}\left(Y_{\psi_{2}}^{2}-\frac{\left(Y_{\psi_{2}}^{e q}\right)^{2}}{\left(Y_{\psi_{1}}^{e q}\right)^{2}} Y_{\psi_{1}}^{2}\right)\right)+\frac{x^{\prime}\left(\frac{g_{* * s}\left(T_{D}\right)}{g_{* s}\left(T_{D}\right)}\right)\left\langle\Gamma_{\eta \rightarrow \overline{\psi_{1}} \psi_{1}}\right\rangle}{H\left(M_{\psi}\right)} Y_{\eta}\right] ; \\
\frac{d Y_{\psi_{2}}}{d x^{\prime}}= & \left(\frac{T^{\prime}}{T}\right)^{2}\left[\frac { s ( M _ { \psi } ) } { x ^ { \prime 2 } H ( M _ { \psi } ) } ( \frac { g _ { * s } ^ { \prime } ( T _ { D } ) } { g _ { * s } ( T _ { D } ) } ) \left(\langle\sigma v\rangle_{e^{+} e^{-} \rightarrow \psi_{2} \psi_{2}}\left(Y_{\psi_{2}}^{e q}\right)^{2}-\langle\sigma v\rangle_{\psi_{2} \psi_{2} \rightarrow Z^{\prime} Z^{\prime}} Y_{\psi_{2}}^{2}\right.\right. \\
& \left.\left.-\langle\sigma v\rangle_{\psi_{2} \psi_{2} \rightarrow \psi_{1} \psi_{1}}\left(Y_{\psi_{2}}^{2}-\frac{\left(Y_{\psi_{2}}^{e q}\right)^{2}}{\left(Y_{\psi_{1}}^{e q}\right)^{2}} Y_{\psi_{1}}^{2}\right)\right)+\frac{x^{\prime}\left(\frac{g_{* s}\left(T_{D}\right)}{\bar{g}_{* s}\left(T_{D}\right)}\right)\left\langle\Gamma_{\eta \rightarrow \overline{\psi_{2}} \psi_{2}}\right\rangle}{H\left(M_{\psi}\right)} Y_{\eta}\right],
\end{aligned}
$$

where $x^{\prime}=\frac{m_{\mathrm{DM}}}{T^{\prime}}=\frac{M_{\psi}}{T^{\prime}}, s\left(M_{\psi}\right)=\frac{2 \pi^{2}}{45} g_{* s} M_{\psi}^{3}$ and $H\left(M_{\psi}\right)=$ $1.67 g_{*}^{1 / 2} \frac{M_{\psi}^{2}}{M_{\mathrm{P} 1}}$. Here, $M_{\psi} \approx M_{1} \approx M_{2}$, ignoring the tiny mass splitting $\Delta m$.

We solve these coupled Boltzmann equations, taking into account the different temperatures of DM and SM sectors after kinetic decoupling, as given in (10). The corresponding evolutions of different comoving number densities are shown in Fig. 9. In Fig. 9, the dot-dashed dark blue line shows the equilibrium number density of the singlet scalar $\eta$ with mass $m_{\eta} \sim 1 \mathrm{TeV}$, which was initially in thermal equilibrium with the SM bath. As its interaction rates falls below the expansion rate, it freezes out, leaving a thermal relic, shown by the green dot-dashed line, assuming it to be stable. The blue dot-dashed line shows the freeze-in production of DM only from the process $e^{+} e^{-} \rightarrow$ DM DM without considering subsequent annihilation of DM into $Z^{\prime}$ pairs. When we take into account both its production from $e^{+} e^{-} \rightarrow \mathrm{DM} \mathrm{DM}$ and subsequent annihilations into $Z^{\prime}$ bosons via $\mathrm{DM} \mathrm{DM} \rightarrow Z^{\prime} Z^{\prime}$, its abundance is depicted by the pink line. The sharp contrast is due to the strong $\mathrm{DM} \mathrm{DM} \rightarrow Z^{\prime} Z^{\prime}$ annihilation rate, which reduces the abundance of DM produced from freeze-in. As the number density of DM increases due

\footnotetext{
${ }^{2}$ This is, to some extent, similar to the super-WIMP dark matter formalism [79].
}

to freeze-in production, the annihilation rate into $Z^{\prime}$ pairs also increases, leading to the first depletion in the pink line around $x=0.1$. Shortly after that, DM production from freeze-in again balances the DM annihilation rate, leading

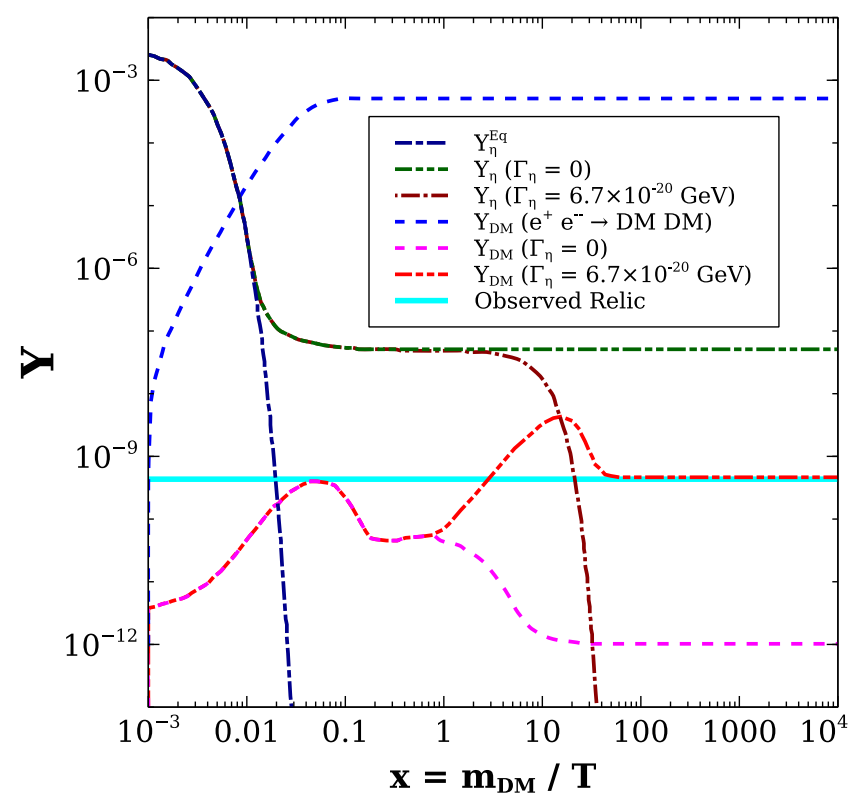

FIG. 9. Comoving number densities of DM and scalar singlet for different cases. 
to a plateau region all the way until $x=1$. However, since freeze-in production from thermal bath becomes negligible beyond $x=1$, we see further depletion in DM density due to its annihilation into $Z^{\prime}$ pairs, leaving an underabundant relic beyond $x=10$. Note that, at this point, we have not considered scalar decay contribution to DM.

Since freeze-in production of DM from the thermal bath followed by DM annihilation into $Z^{\prime}$ pairs lead to underabundant relic density, we now consider the additional contribution from scalar singlet decay. The red dot-dashed line shows the evolution of comoving number density of DM after taking the scalar decay contribution into account. The corresponding evolution of the scalar number density is shown by the maroon-colored dot-dashed line. Clearly, once the number density of the scalar falls due to its decay, the DM number density gets uplifted. Once the decay is complete, DM relic also saturates beyond $x \approx 30$. It should be noted that the scalar decay occurs after DM annihilation to $Z^{\prime}$ pairs freezes out around $x=10$ to avoid further depletion. Also, while considering freeze-in production of DM from the thermal bath, we considered the contribution of electron-positrons only, for simplicity. If we consider all the particles in the thermal bath, we get more freeze-in production of DM, and the final required abundance of DM can be realized by appropriate tuning of scalar decay width without affecting rest of the analysis related to selfinteraction and XENON1T excess.

Note that the lines showing the evolution of DM number density in Fig. 9 considers both the DM components $\psi_{1,2}$. Since their mass splitting is very small, $\Delta m \sim \mathcal{O}(\mathrm{keV})$, they behave very similarly as far as calculation of relic abundance goes. However, once the net relic is generated, there can be interconversion between two DM components dominantly through $Z^{\prime}$-mediated t-channel process $\psi_{2} \psi_{2} \rightarrow \psi_{1} \psi_{1}$. We take this into account and show that the effect of such interconversion with such small mass splitting $\left(\Delta m=2 \times 10^{-6} \mathrm{GeV}\right)$ is negligible. This can be seen from Fig. 10, where the fractional contributions $Y_{\mathrm{DM}_{1}} / Y_{\mathrm{DM}_{\text {Total }}}$ and $Y_{\mathrm{DM}_{2}} / Y_{\mathrm{DM}_{\text {Total }}}$ for mass splitting $\Delta m=$ $2 \times 10^{-6} \mathrm{GeV}$ are shown. We have also taken into account the Sommerfeld effect induced by the multiple $Z^{\prime}$ boson exchange in the inter-conversion process [80]. Clearly, such interconversions lead to negligible effects on individual DM relic abundance, and hence we consider them to be equally dominant in the rest of our analysis.

\section{XENON1T EXCESS}

The direct detection prospects of such self-interacting DM can be addressed through the recently reported excess in the electron recoil events at the XENON1T experiment. The DM-electron scattering is shown in Fig. 11. We assume $\psi_{2}$ is heavier than $\psi_{1}$ with a small mass splitting $\Delta m=$ $M_{2}-M_{1}$ between the two components. Because of this

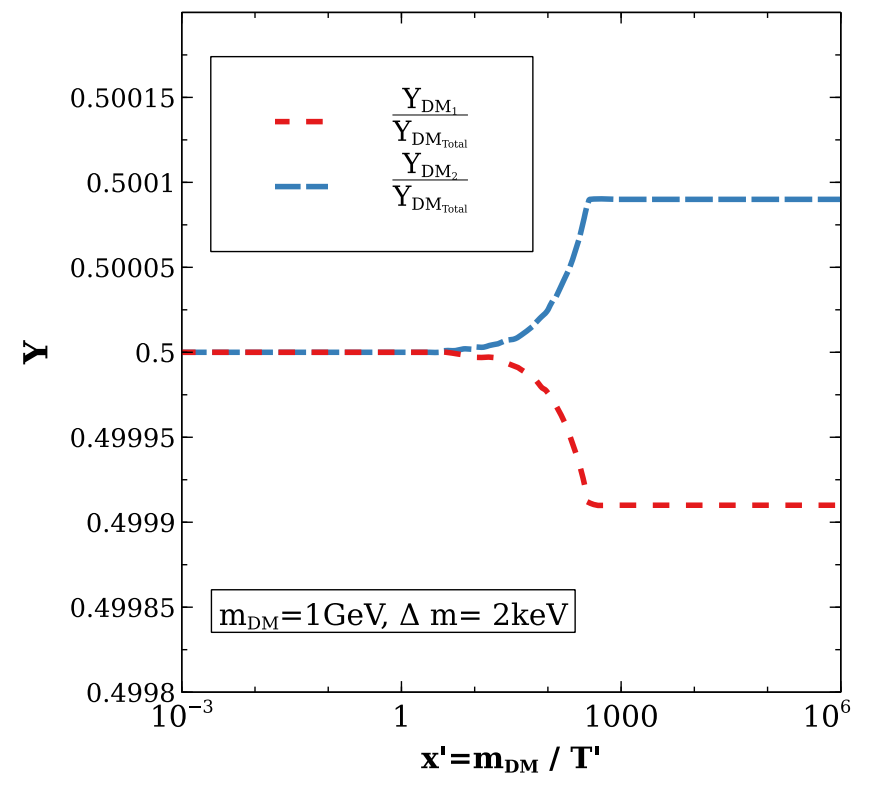

FIG. 10. Fractional contributions $Y_{\mathrm{DM}_{1}} / Y_{\mathrm{DM}_{\text {Total }}}$ and $Y_{\mathrm{DM}_{2}} / Y_{\mathrm{D}_{\text {Total }}}$ to DM relic density for $\Delta m=2 \times 10^{-6} \mathrm{GeV}$.

inelastic nature of these DM candidates and since the mass splitting $\Delta m$ is kept fixed at the $\mathrm{keV}$ scale, we can successfully explain the recently reported XENON1T anomaly [28]. For a fixed incoming velocity $v$ of heavier DM $\psi_{2}$, the differential scattering cross section for the down-scattering process $\psi_{2} e \rightarrow \psi_{1} e$ (with electrons inside the Xenon atom) can be written as

$$
\frac{d\langle\sigma v\rangle}{d E_{r}}=\frac{\sigma_{e}}{2 m_{e} v} \int_{q^{-}}^{q+} a_{0}^{2} q d q|F(q)|^{2} K\left(E_{r}, q\right),
$$

where $m_{e}$ is the electron mass, $\sigma_{e}$ is the corresponding free electron cross section at fixed momentum transfer $q=$ $1 / a_{0}$ with $a_{0}=\frac{1}{\alpha m_{e}}$ being the Bohr radius and $\alpha=\frac{e^{2}}{4 \pi}=\frac{1}{137}$ being the fine structure constant, $E_{r}$ is the recoil energy of electron, and $K\left(E_{r}, q\right)$ is the atomic excitation factor. For our calculations, the atomic excitation factor is adopted from Ref. [81]. We assume the DM form factor to be unity.

However, to include velocity dispersion in Eq. (13), we use the distribution function (obtained after angular integration of a Maxwellian velocity distribution boosted in Earth's rest frame)

$$
f(v)=A v \operatorname{Exp}\left[-3\left(v-v_{m}\right)^{2} / 2 \sigma_{v}^{2}\right],
$$

where $A$ is the normalization constant such that $\int f(v) d v=1$. The details of velocity distribution are given in Appendix A 3. In Eq. (14), $v_{m}$ is the most-probable velocity of DM, which is induced by the relative velocity of the Sun with respect to the Galactic halo. Here, $\sigma_{v}$ is the $\mathrm{DM}$ velocity dispersion, which is given by $\sigma_{v}^{2}=\frac{3}{2} v_{m}^{2}$. As a result, Eq. (13), after incorporating the velocity dispersion of DM, can be rewritten as $[38,51,81,82]$ 


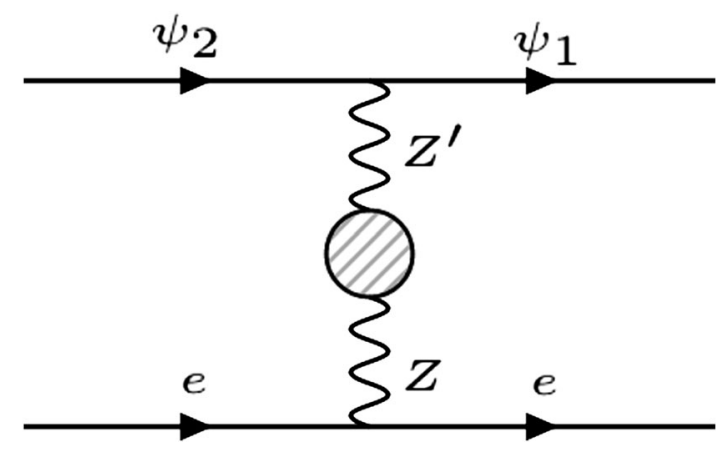

FIG. 11. DM-electron scattering at XENON1T.

$\frac{d\langle\sigma v\rangle}{d E_{r}}=\frac{\sigma_{e}}{2 m_{e}} \int_{0}^{v_{\mathrm{esc}}} d v \frac{f(v)}{v} \int_{q-}^{q+} a_{0}^{2} q d q|F(q)|^{2} K\left(E_{r}, q\right)$,

where $v_{\text {esc }}$ is the DM escape velocity in the Milky Way, which is of the order $v_{\text {esc }} \sim 533_{-41}^{+54} \mathrm{~km} / \mathrm{s}$ [83]. In inelastic DM scenarios, the minimum DM velocity $\left(v_{\min }\right)$ required by the DM to up-scatter to the Next-to-lightest stable particle (NLSP) and register a recoil inside the detector is decided by the kinematics of scattering. However, it is worth mentioning that in the case of an inelastic downscattering of DM with an electron, which we consider here, there is no kinematic limit on the minimum velocity of DM as the incoming particle with almost vanishing velocity can still down-scatter to the lighter component with the mass splitting between the DM components being transferred to the electron recoil energy, without violating anything kinematically.

The free electron scattering cross section for the process $\psi_{2} e \rightarrow \psi_{1} e$ is given by

$$
\sigma_{e}=\frac{16 \pi \alpha_{Z} \alpha^{\prime} \epsilon^{2} m_{e}^{2}}{M_{Z^{\prime}}^{4}}
$$

where $\alpha_{Z}=\frac{g^{2}}{4 \pi}, \quad \alpha^{\prime}=\frac{g^{\prime 2}}{4 \pi}$ and $\epsilon$ is the kinetic mixing parameter between $Z$ and $Z^{\prime}$ gauge bosons. For chosen

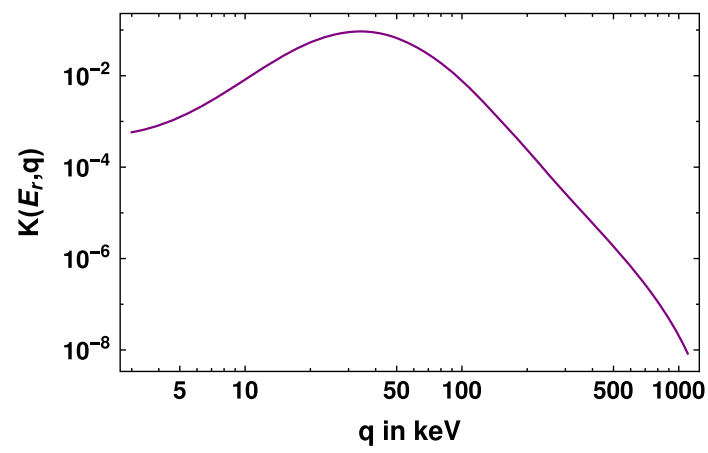

values of DM and mediator masses in our work, this kinetic mixing is required to be $\epsilon \sim 10^{-8}$. It should be noted that, for $\mathrm{GeV}$-scale $\mathrm{DM}, \sigma_{e}$ is independent of DM mass as the reduced mass of the DM-electron is almost equal to electron mass. The limits of integration for the inelastic scattering in Eq. (15) are determined depending on the relative values of recoil energy $\left(E_{r}\right)$ and the mass splitting between the two DM components.

For $E_{r} \geq \Delta m$,

$$
q_{ \pm}=M_{2} v \pm \sqrt{M_{2}^{2} v^{2}-2 M_{2}\left(E_{r}-\Delta m\right)},
$$

and for $E_{r} \leq \Delta m$,

$$
q_{ \pm}=\sqrt{M_{2}^{2} v^{2}-2 M_{2}\left(E_{r}-\Delta m\right)} \pm M_{2} v .
$$

The dependency of the atomic excitation factor on the momentum transferred $q$ is shown in Fig. 12. Here, the dominant contribution comes from the bound states with principal quantum number $n=3$ as their binding energy is around a few keV. In the right panel of Fig. 12, we show the plot for the integration of momentum transferred times the atomic excitation factor [i.e., $K_{\text {int }}\left(E_{r}, q\right)=\int_{q-}^{q+} q d q K\left(E_{r}, q\right)$ ] as a function of the recoil energy $E_{r}$ for $M_{1}=0.3 \mathrm{GeV}$ and $\Delta m=2 \mathrm{keV}$. The figure shows a peak around $E_{r} \simeq \Delta m$ since the $q_{-}$approaches to zero and the momentum transfer maximizing this factor is available. It is worth mentioning that such a kind of enhancement is a characteristic feature of inelastic scattering.

The differential event rate for the inelastic DM scattering with electrons in the xenon atom, i.e., $\psi_{2} e \rightarrow \psi_{1} e$, can be given as

$$
\frac{d R}{d E_{r}}=n_{T} n_{\mathrm{DM}} \frac{d\langle\sigma v\rangle}{d E_{r}},
$$

where $n_{T}=4 \times 10^{27} \mathrm{Ton}^{-1}$ is the number density of xenon atoms and $n_{\mathrm{DM}}$ is the number density of the dark matter particle.

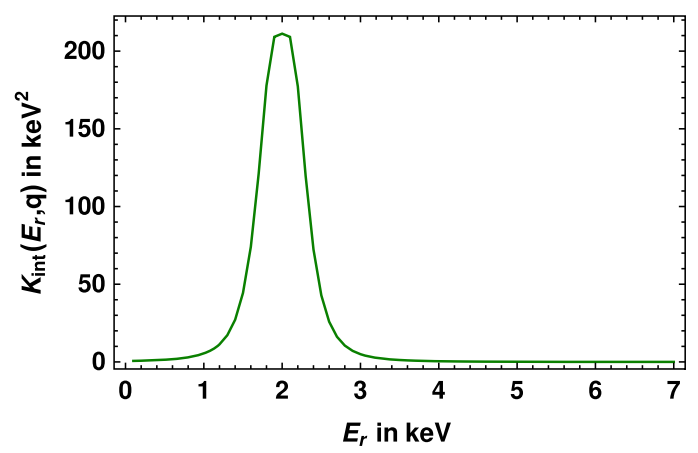

FIG. 12. Left panel: Atomic excitation factor is shown as a function of momentum transferred. Right panel: The atomic excitation factor after being integrated over the transferred momentum is shown as a function of the transferred recoil energy $E_{r}$. 
The detected recoil energy spectrum can be obtained by convolving Eq. (19) with the energy resolution of the XENON1T detector. Incorporating the detector efficiency $\gamma(E)$, the energy resolution of the detector is given by a Gaussian distribution with an energy-dependent width,

$$
\zeta\left(E, E_{r}\right)=\frac{1}{\sqrt{2 \pi \sigma_{\mathrm{det}}^{2}}} \operatorname{Exp}\left[-\frac{\left(E-E_{r}\right)^{2}}{2 \sigma_{\mathrm{det}}^{2}}\right] \times \gamma(E),
$$

where $\gamma(E)$ is reported in Fig. 2 of Ref. [28] and the width $\sigma_{\text {det }}$ is given by

$$
\sigma_{\mathrm{det}}(E)=a \sqrt{E}+b E
$$

with $a=0.3171$ and $b=0.0037$. Thus, the final detected recoil energy spectrum is given by

$$
\begin{aligned}
\frac{d R_{\mathrm{det}}}{d E_{r}}= & \frac{n_{T} n_{\mathrm{DM}} \sigma_{e} a_{0}^{2}}{2 m_{e}} \int d E \zeta\left(E, E_{r}\right) \\
& \times\left[\int_{0}^{v_{\mathrm{esc}}} d v \frac{f(v)}{v} \int_{q-}^{q+} d q q K\left(E_{r}, q\right)\right] .
\end{aligned}
$$

To obtain the fit shown in the upper panel of Fig. 13, the mass splitting is taken to be $\Delta m=2 \mathrm{keV}$, while heavier DM mass is taken to be $1 \mathrm{GeV}$. The other relevant
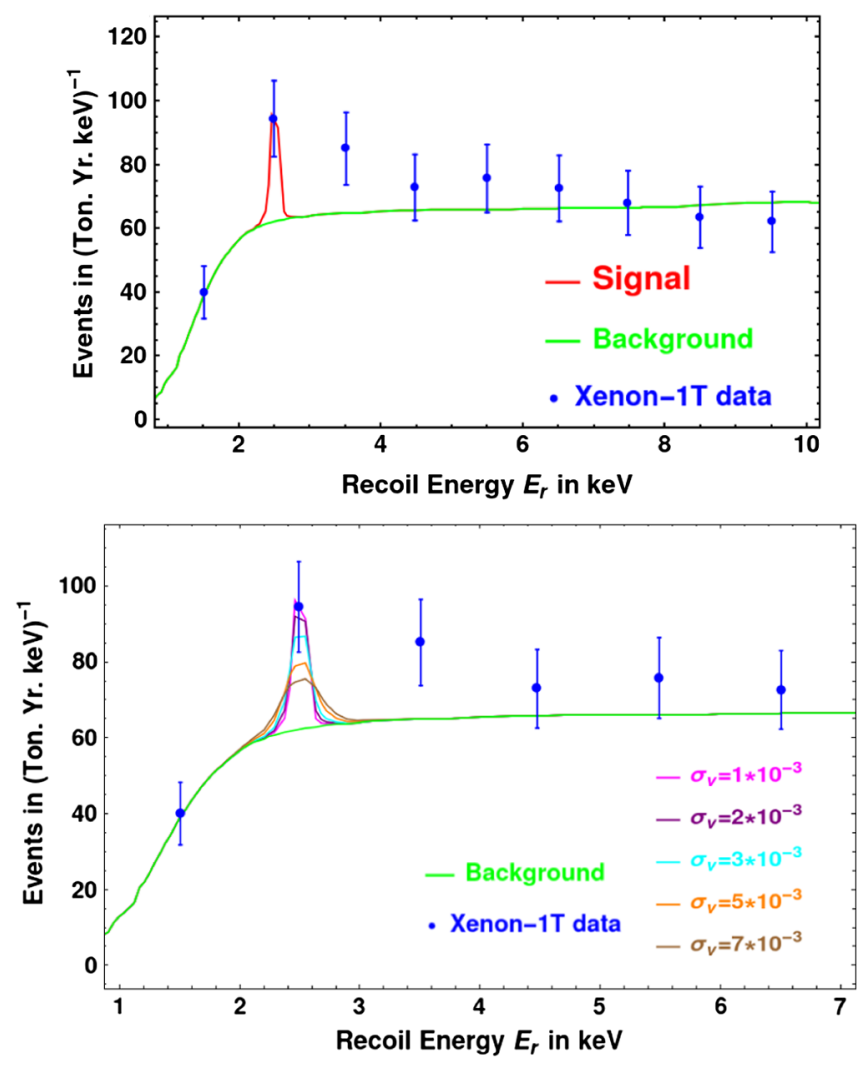

FIG. 13. Fit to XENON1T electron recoil excess with the selfinteracting inelastic DM in our model. parameters used in this fit are $\sigma_{v}=\sqrt{3 / 2} v_{m}$ with $v_{m}=$ $1 \times 10^{-3}, \quad g^{\prime}=0.1, \quad M_{Z^{\prime}}=10 \mathrm{MeV}$, and $\epsilon=4 \times 10^{-8}$, which corresponds to cross section $\sigma_{e}=1.9 \times 10^{-17} \mathrm{GeV}^{-2}$.

On the other hand, in the bottom panel of Fig. 13, we show the fit considering different velocity dispersion for the DM particle as we have no observational constraints on $f(v)$ apart from numerical simulations. Clearly, as we increase the velocity dispersion, the peak in the spectrum giving an appreciable fit gets flattened out and no longer explains the XENON1T signal within $E_{r}=2-3 \mathrm{keV}$ for larger $\sigma_{v}$.

\section{CONCLUSION}

We summarize our key findings in Fig. 14. We show all the relevant constraints as well as favored parameter space in the $g^{\prime}-M_{Z^{\prime}}$ plane. In Fig. 14, all the colored regions (except the blue region which corresponds to XENON1T excess) represent disfavored regions from different bounds. The green patch represents the region where the DM selfscattering cross section is not large enough to solve the astrophysical problems discussed in Sec. III. To be more quantitative, the green shaded regions correspond to the DM self-scattering cross section $\sigma / m<0.1 \mathrm{~cm}^{2} / \mathrm{g}$. The triangular region on the upper left corner of Fig. 14 is disfavored from the lower bound on the lifetime of heavier DM. Since the mass splitting between $\psi_{1}$ and $\psi_{2}$ is kept at

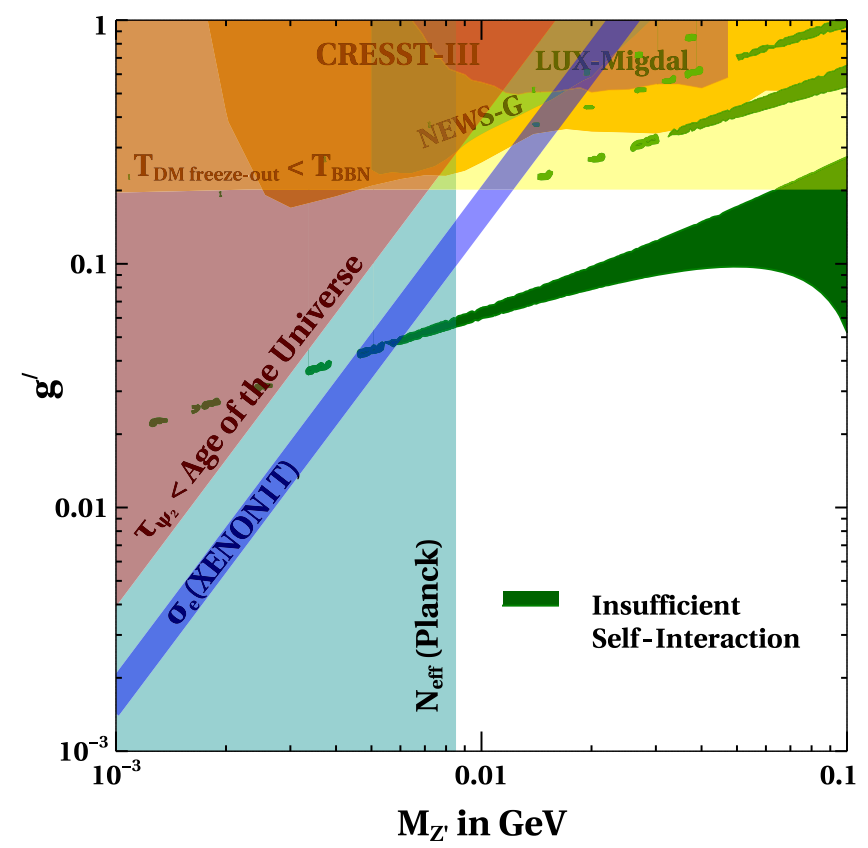

FIG. 14. Summary plot for inelastic self-interacting DM showing the final parameter space from relevant constraints. The white region represents the allowed parameter space available after imposing all the constraints. The blue patch represents the parameter space allowed by XENON1T for $1 \mathrm{GeV}$ inelastic DM with mass splitting $\Delta m=2 \mathrm{keV}$ and kinetic mixing parameter $\epsilon=4 \times 10^{-8}$. 
the $\mathrm{keV}$ scale $\Delta m=\mathcal{O}(\mathrm{keV})$, there can be decay modes like $\psi_{2} \rightarrow \psi_{1} \nu \bar{\nu}$ mediated by $Z-Z^{\prime}$ mixing. If both the DM components are to be there in the present Universe, this lifetime has to be more than the age of the Universe, that is, $\tau_{\psi_{2}}>\tau_{\text {Univ. }}$. The decay width of this process is $\Gamma\left(\psi_{2} \rightarrow \psi_{1} \nu \bar{\nu}\right)=\frac{g^{2} g^{2} \epsilon^{2}(\Delta m)^{5}}{160 \pi^{3} M_{Z^{\prime}}^{4}}$. Thus, imposing the lifetime constraint on heavier DM, we get the triangular shaded region. We also show the parameter space excluded by the recent results from CRESST-III [84], LUX-Migdal [85], and NEWS-G [86] on low mass DM. This corresponds to the shaded region of orange, brown, and light green color in the topmost part of Fig. 14. The bound from EDELWEISSIII [87] is much weaker than the above-mentioned experiments. Assuming $M_{Z^{\prime}}=0.01 M_{\mathrm{DM}}$, these are the only experiments that are sensitive to the parameter space we are interested in. We have checked that the constraints from other low-threshold experiments like DAMIC, PICO, PANDAX-II, CDMSlite, etc., do not apply to our parameter space. The solid band of blue color corresponds to the free electron cross section $\sigma_{e}=(1-5) \times 10^{-17} \mathrm{GeV}^{-2}$, which is required to obtain the fit for the XENON1T excess for a DM of mass around $1 \mathrm{GeV}$ with a typical DM velocity of order $\mathcal{O}\left(10^{-3}\right)$. The shaded region of yellow color at the top corresponds to the region where DM annihilation into $Z^{\prime}$ pairs does not freeze out before the epoch of big bang nucleosynthesis. This will require scalar singlet decay at post-big bang nucleosynthesis epochs. Additionally the $Z^{\prime}$ bosons which keep getting produced from DM annihilations will decay into light SM fermions, injecting new relativistic degrees of freedom. Since all these may potentially ruin the successful predictions of the big bang nucleosynthesis, we disfavor this region of parameter space. Since our chosen value of kinetic mixing is very small, the flavor bounds on such light $Z^{\prime}$ bosons from dark photon searches at $B A B A R$ [88] are automatically satisfied. Additionally, CMB bounds from Planck measurements on DM annihilations into charged fermions [1] are trivially satisfied as all such processes remain suppressed by kinetic mixing. Another constraint on the parameter space arises due to late decay of $Z^{\prime}$ into SM leptons. For example, if $Z^{\prime}$ decays after neutrino decoupling temperature $T_{\mathrm{dec}}^{\nu} \sim \mathcal{O}(\mathrm{MeV})$, it will increase the effective relativistic degrees of freedom is tightly constrained by Planck. 2018 data as $N_{\text {eff }}=2.99_{-0.33}^{+0.34}$ [1]. As pointed out by the authors of Ref. [89], such constraints can be satisfied if $M_{Z^{\prime}} \gtrsim$ 8.5 MeV for the chosen value of kinetic mixing parameter in our work. We show this as the light green shaded region toward the left in Fig. 14. Note that we have not imposed any constraints from the DM relic point of view as that can be satisfied independently by appropriate tuning of scalar singlet parameters discussed before.

To conclude, we have studied the possibility of selfinteracting DM as a possible explanation of the recently reported XENON1T excess. While XENON1T excess can arise due to inelastic nature of DM so that the heavier DM can undergo a down-scattering with electrons, the corresponding mediator of such scattering, if sufficiently light compared to DM, can also give rise to the required selfinteraction cross section $\sigma / m$ required to solve the smallscale structure problems associated with cold dark matter. We consider a hidden $U(1)_{X}$ gauge symmetry under which the inelastic DM is charged and this dark sector interacts with the SM purely via kinetic mixing of $U(1)_{X}$ with $U(1)_{Y}$ of the standard model. The requirement of large selfinteraction or $U(1)_{X}$ gauge coupling forces us to consider tiny kinetic mixing required to generate the XENON1T excess while satisfying all other experimental bounds. This tiny kinetic mixing also prevents DM from reaching chemical equilibrium with the SM requiring its nonthermal or freeze-in production from the SM bath. However, because of the large coupling of DM with the $U(1)_{X}$ gauge boson $Z^{\prime}$, the DM particles can annihilate strongly into much lighter $Z^{\prime}$ bosons, depleting the number density generated from freeze-in. To fill the gap, we introduce another long-lived scalar singlet which freezes out from the thermal bath and decays very late into $\mathrm{DM}$, generating the required relic. As seen from the summary plot in Fig. 14, after applying all relevant bounds, there exists only a tiny parameter space (the blue shaded region not overlapped with other regions) that can give rise to the required XENON1T excess and DM self-interactions for $1 \mathrm{GeV}$ inelastic DM with mass splitting of $2 \mathrm{keV}$, while being consistent with all other bounds. Future data from XENON1T experiment as well as other searches should be able to further constrain or confirm this predictive scenario.

Now, we turn to comment on the implications of thermally generated self-interacting dark matter $\psi_{1}$ and $\psi_{2}$, which we assume to constitute about $1 \%$ of the total relic (see Fig. 7). Since the relic density is smaller by 2 orders of magnitude than the observed one, the corresponding DM-electron cross section $\sigma_{e}\left(\psi_{2} e \rightarrow \psi_{1} e\right)$ has to be increased by two orders in order to explain the observed XENON1Texcess. This can be achieved by increasing $\epsilon$ by 1 order of magnitude, since $\sigma_{e} \propto \epsilon^{2}$. However, increasing $\epsilon$ by 1 order of magnitude will not satisfy the lifetime bound on $\psi_{2}$ as $\tau_{\psi_{2}} \propto 1 / \epsilon^{2}$. Note that such subdominant SIDM will also not solve the small-scale structure problem, even if the DM deficit is filled by some other component which is connected to neither SIDM nor to the observed XENON1T excess.

\section{ACKNOWLEDGMENTS}

D. B. acknowledges the support from Early Career Research Award from DST-SERB, Government of India (Grant No. ECR/2017/001873). M. D. acknowledges Department of Science and Technology (DST), Government of India, for providing the financial assistance for the research under Grant No. DST/INSPIRE/03/2017/ 000032. M.D. would also like to acknowledge Tracy R. Slatyer and Katelin Schutz for useful discussion regarding self-interating dark matter. 


\section{APPENDIX: RELEVANT CROSS SECTION AND DECAY WIDTHS}

\section{Self-interaction cross sections at low energy}

The scattering cross sections can be derived as [68]

$$
\begin{gathered}
\sigma_{\psi_{1} \psi_{1} \rightarrow \psi_{1} \psi_{1}}=\frac{\pi}{\epsilon_{v}^{2}}\left|1+\left(\frac{V_{0}}{4 \mu^{2}}\right)^{-\frac{2 i \epsilon_{v}}{\mu}}\left(\frac{\Gamma_{v}}{\Gamma_{v}^{*}}\right)\left[\frac{\cosh \left(\frac{\pi\left(\epsilon_{\Delta}+\epsilon_{v}\right)}{2 \mu}\right) \sinh \left(\frac{\pi\left(\epsilon_{v}-\epsilon_{\Delta}\right)}{2 \mu}+i \varphi\right)}{\cosh \left(\frac{\left.\pi\left(\epsilon_{\Delta}-\epsilon_{v}\right)\right)}{2 \mu} \sinh \left(\frac{\pi\left(\epsilon_{v}+\epsilon_{\Delta}\right)}{2 \mu}-i \varphi\right)\right.}\right]\right|^{2} \\
\sigma_{\psi_{2} \psi_{2} \rightarrow \psi_{2} \psi_{2}}=\frac{\pi}{\epsilon_{\Delta}^{2}}\left|1+\left(\frac{V_{0}}{4 \mu^{2}}\right)^{-\frac{2 i \epsilon_{\Delta}}{\mu}}\left(\frac{\Gamma_{\Delta}}{\Gamma_{\Delta}^{*}}\right)\left[\frac{\cosh \left(\frac{\pi\left(\epsilon_{\Delta}+\epsilon_{v}\right)}{2 \mu}\right) \sinh \left(\frac{\pi\left(\epsilon_{v}-\epsilon_{\Delta}\right)}{2 \mu}+i \varphi\right)}{\cosh \left(\frac{\left.\pi\left(\epsilon_{\Delta}-\epsilon_{v}\right)\right)}{2 \mu} \sinh \left(\frac{\pi\left(\epsilon_{v}+\epsilon_{\Delta}\right)}{2 \mu}-i \varphi\right)\right.}\right]\right|^{2} \\
\sigma_{\psi_{1} \psi_{1} \rightarrow \psi_{2} \psi_{2}}=\frac{2 \pi \cos ^{2} \varphi \sinh \left(\frac{\left.\pi \epsilon_{v}\right)}{\mu}\right) \sinh \left(\frac{\pi \epsilon_{\Delta}}{\mu}\right)}{\epsilon_{v}^{2} \cosh ^{2}\left(\frac{\pi\left(\epsilon_{\Delta}-\epsilon_{v}\right)}{2 \mu}\right)\left(\cosh \left(\frac{\pi\left(\epsilon_{v}+\epsilon_{\Delta}\right)}{\mu}\right)-\cosh (2 \varphi)\right)} \\
\sigma_{\psi_{2} \psi_{2} \rightarrow \psi_{1} \psi_{1}}=\frac{2 \pi \cos ^{2} \varphi \sinh \left(\frac{\left.\pi \epsilon_{v}\right)}{\mu}\right) \sinh \left(\frac{\pi \epsilon_{\Delta}}{\mu}\right)}{\epsilon_{\Delta}^{2} \cosh ^{2}\left(\frac{\pi\left(\epsilon_{\Delta}-\epsilon_{v}\right)}{2 \mu}\right)\left(\cosh \left(\frac{\pi\left(\epsilon_{v}+\epsilon_{\Delta}\right)}{\mu}\right)-\cosh (2 \varphi)\right)}
\end{gathered}
$$

where we have defined $\epsilon_{\Delta}=\sqrt{\epsilon_{v}^{2}-\epsilon_{\delta}^{2}}$ and $\mu$ and $V_{0}$ are defining parameters for the exponential potential $V_{0} e^{-\mu r}$, given by

$$
\mu=\epsilon_{Z}\left(\frac{1}{2}+\frac{1}{2} \sqrt{1+\frac{4}{\epsilon_{Z} r_{M}}}\right), \quad V_{0}=\frac{e^{\epsilon_{Z} r_{M}\left(-\frac{1}{2}+\frac{1}{2} \sqrt{1+\frac{4}{\epsilon_{Z} r_{M}}}\right.}}{r_{M}}
$$

Here, $r_{M}$ is chosen from the relation $e^{-\epsilon_{\phi} r_{M}} / r_{M}=\max \left(\epsilon_{\delta}^{2} / 2, \epsilon_{\phi}^{2}\right)$. The terms $\Gamma_{v}$ and $\Gamma_{\Delta}$ are given by

$$
\begin{gathered}
\Gamma_{v}=\Gamma\left(1+i \frac{\epsilon_{v}}{\mu}\right) \Gamma\left(i \frac{\epsilon_{v}-\epsilon_{\Delta}}{2 \mu}+\frac{1}{2}\right) \Gamma\left(i \frac{\epsilon_{v}+\epsilon_{\Delta}}{2 \mu}+\frac{1}{2}\right) \\
\Gamma_{\Delta}=\Gamma\left(1+i \frac{\epsilon_{\Delta}}{\mu}\right) \Gamma\left(i \frac{\epsilon_{\Delta}-\epsilon_{v}}{2 \mu}+\frac{1}{2}\right) \Gamma\left(i \frac{\epsilon_{v}+\epsilon_{\Delta}}{2 \mu}+\frac{1}{2}\right),
\end{gathered}
$$

with $\Gamma$ denoting the gamma function.

\section{Interactions for DM relic calculations}

$$
\begin{aligned}
\sigma\left(\mathrm{DM} \mathrm{DM} \rightarrow e^{+} e^{-}\right)= & \frac{g^{2} g^{\prime 2} \epsilon^{2}\left(2 s+\left(M_{\psi_{1}}+M_{\psi_{2}}\right)^{2}\right) \sqrt{M_{\psi_{1}}^{4}+\left(s-M_{\psi_{2}}^{2}\right)^{2}-2 M_{\psi_{1}}^{2}\left(s+M_{\psi_{2}}^{2}\right)}}{192 \pi \cos ^{2} \theta_{W}\left(s-M_{Z^{\prime}}^{2}\right)^{2}\left(s-\left(M_{\psi_{1}}+M_{\psi_{2}}\right)^{2}\right)} \\
\sigma\left(\mathrm{DM} \mathrm{DM} \rightarrow Z^{\prime} Z^{\prime}\right) \simeq & \frac{g^{\prime 4}}{192 \pi M_{Z^{\prime}}^{4} s\left(s-4 M_{\psi}^{2}\right)} \times\left[\frac{24 M_{Z^{\prime}}^{4} s\left(4 m_{\psi}^{4}+2 M_{Z^{\prime}}^{4}+s M_{\psi}^{2}\right) A}{M_{Z^{\prime}}^{4}+M_{\psi}^{2}\left(s-4 M_{Z^{\prime}}^{2}\right)}\right. \\
& \left.-\frac{24 M_{Z^{\prime}}^{4}\left(8 M_{\psi}^{2}-4 M_{Z^{\prime}}^{2}-s^{2}-\left(s-2 M_{Z^{\prime}}^{2}\right) 4 M_{\psi}^{2}\right)}{s-2 M_{Z^{\prime}}^{2}} \log \left[\frac{2 M_{Z^{\prime}}^{2}+s(A-1)}{2 M_{Z^{\prime}}^{2}-s(A+1)}\right]\right],
\end{aligned}
$$

where $A=\sqrt{\frac{\left(s-4 M_{Z^{\prime}}^{2}\right)\left(s-4 M_{\psi_{1}}^{2}\right)}{s^{2}}}$ 


$$
\begin{aligned}
\sigma\left(e^{+} e^{-} \rightarrow \mathrm{DM} \mathrm{DM}\right) & =\frac{g^{2} g^{\prime 2} \epsilon^{2}\left(s+2 M_{\psi}^{2}\right)\left(s-M_{e}^{2}-4\left(s+2 M_{e}^{2}\right) \sin ^{2} \theta_{W}\right)}{96 \pi \cos ^{2} \theta_{W}\left(s-4 M_{e}^{2}\right)\left(s-M_{Z^{\prime}}^{2}\right)^{2}} \sqrt{\frac{\left(s-4 M_{e}^{2}\right)\left(s-4 M_{\psi}^{2}\right)}{s^{2}}} \\
\sigma\left(\mathrm{DM} e^{-} \rightarrow \mathrm{DM} e^{-}\right) & =\frac{g^{2} g^{\prime 2} \epsilon^{2} A}{128 \pi \cos ^{2} \theta_{W} m_{Z^{\prime}}^{4}\left(s-M_{e}^{2}-M_{\psi_{2}}^{2}\right)^{2}} \frac{B}{C}-D \log \left[\frac{E+s\left(2 M_{Z^{\prime}}^{2}-M_{\psi_{2}}^{2}+s+A\right)}{E+s\left(2 M_{Z^{\prime}}^{2}-M_{\psi_{2}}^{2}+s-A\right)}\right]
\end{aligned}
$$

where

$$
\begin{aligned}
A= & s\left(\frac{\left(M_{e}^{4}+\left(M_{\psi_{1}}^{2}-s\right)^{2}-2 M_{e}^{2}\left(M_{\psi_{1}}^{2}+s\right)\right)\left(M_{e}^{4}+\left(M_{\psi_{2}}^{2}-s\right)^{2}-2 M e^{2}\left(M_{\psi_{2}}^{2}+s\right)\right)}{s^{4}}\right)^{\frac{1}{2}} \\
B= & s\left(2 M_{Z^{\prime}}^{4} s+2\left(M_{\psi_{1}}^{2}-s\right)\left(M_{\psi_{2}}^{2}-s\right) s+M_{e}^{4}\left(M_{Z^{\prime}}^{2}+2 s\right)+M_{Z^{\prime}}^{2}\left(M_{\psi_{1}}^{2} M_{\psi_{2}}^{2}-2\left(M_{\psi_{1}}^{2}-M_{\psi_{1}} M_{\psi_{2}}+M_{\psi_{2}}^{2}\right) s+3 s^{2}\right)\right) \\
& +M_{e}^{2}\left(\left(M_{\psi_{1}}^{2}-M_{\psi_{2}}^{2}\right)^{2}+4 M_{\psi_{1}} M_{\psi_{2}} s-4 s^{2}-M_{Z^{\prime}}^{2}\left(M_{\psi_{1}}^{2}+M_{\psi_{2}}^{2}+2 s\right)\right) \\
C= & M_{e}^{4} M_{Z^{\prime}}^{2}+M_{Z^{\prime}}^{2}\left(\left(M_{\psi_{1}}^{2}-s\right)\left(M_{\psi_{2}}^{2}-s\right)+M_{Z^{\prime}}^{2} s\right)+M_{e}^{2}\left(\left(M_{\psi_{1}}^{2}-M_{\psi_{2}}^{2}\right)^{2}-M_{Z^{\prime}}^{2}\left(M_{\psi_{1}}^{2}+M_{\psi_{2}}^{2}+2 s\right)\right) \\
D= & 2 M_{Z^{\prime}}^{2}-\left(M_{\psi_{2}}-M_{\psi_{1}}\right)^{2}+2 s \\
E= & M_{e}^{4}+M_{\psi_{1}}^{2}\left(M_{\psi_{2}}^{2}-s\right)-M_{e}^{2}\left(M_{\psi_{1}}^{2}+M_{\psi_{2}}^{2}+2 s\right)
\end{aligned}
$$

$$
\sigma\left(\eta^{\dagger} \eta \rightarrow H^{\dagger} H\right)=\frac{\lambda}{16 \pi s} \sqrt{\frac{s-4 M_{\eta}^{2}}{s-4 M_{H}^{2}}} .
$$

The decay width of the scalar singlet $\eta$ is given by

$$
\Gamma(\eta \rightarrow D M D M)=\frac{\lambda^{2}}{8 \pi} m_{\eta}\left(1-4 \frac{m_{\mathrm{DM}}^{2}}{m_{\eta}^{2}}\right)^{3 / 2} .
$$

The decay width of $Z^{\prime}$ is given by

$$
\Gamma\left(Z^{\prime} \rightarrow \overline{f f}\right)=\frac{\epsilon^{2} g^{2} M_{Z^{\prime}}}{48 \pi \cos ^{2} \theta_{W}}\left(C_{V_{f}}^{2}+C_{A_{f}}^{2}\right) .
$$

The thermal average cross section is given by [90]

$$
\begin{aligned}
\langle\sigma v\rangle_{C M}= & \frac{x}{2\left[K_{1}^{2}(x)+K_{2}^{2}(x)\right]} \\
& \times \int_{2}^{\infty} d z \sigma\left(z^{2} m_{\psi}^{2}\right)\left(z^{2}-4\right) z^{2} K_{1}(z x) .
\end{aligned}
$$

\section{DM velocity distribution function}

The distribution function used in Eq. (14) can be obtained as follows. Let $\vec{u}$ and $\vec{v}$ be the velocities of dark matter in the rest frames of the Galaxy and Earth, respectively. If $\overrightarrow{v_{E}}$ is the velocity of Earth with respect to the Galactic rest frame, then we have $\vec{u}=\vec{v}+\overrightarrow{v_{E}}$. Assuming that the velocity distribution of dark matter with respect to the Galactic rest frame is Maxwellian, we can write

$$
f(\vec{u}) d^{3} u=N e^{\frac{-3|\vec{u}|^{2}}{2 \sigma^{2}}} d^{3} u=N e^{\frac{-3\left(\overrightarrow{v_{E}}+\vec{v}\right)^{2}}{2 \sigma_{v}^{2}}} d^{3} v,
$$

where $N$ is the normalization constant and $\sigma_{v}$ is the velocity dispersion. Assuming spherical symmetry and considering the $z$ axis in the direction of $\overrightarrow{v_{E}}$ which subtends an angle $\theta$ with $\vec{v}$, we can write

$$
N e^{\frac{-3\left(\overrightarrow{v_{E}}+\vec{v}\right)^{2}}{2 \sigma_{v}^{2}}} d^{3} v=N v^{2} d v d \phi d \cos \theta e^{\frac{-3\left(v_{E}^{2}+v^{2}+2 v_{E} v \cos \theta\right)}{2 \sigma_{v}^{2}}} .
$$

Now, carrying out the integration for the angular coordinates $\phi$ and $\theta$, we obtain

$$
\begin{aligned}
f(v) d v & =N 2 \pi v^{2} d v e^{\frac{-3\left(v_{E}^{2}+v^{2}\right)}{2 \sigma_{v}^{2}}} \int d \cos \theta e^{\frac{-3 v_{E} v \cos \theta}{\sigma_{v}^{2}}} \\
& =N 2 \pi v^{2} d v e^{\frac{-3\left(v_{E}^{2}+v^{2}\right)}{2 \sigma_{v}^{2}}} \frac{\sigma_{v}^{2}}{3 v_{E} v}\left[e^{3 \frac{v_{E} v}{\sigma_{v}^{2}}}-e^{-3 \frac{v_{E} v}{\sigma_{v}^{2}}}\right] \\
& \simeq N 2 \pi \frac{\sigma_{v}^{2}}{3 v_{E}} v d v e^{-3 \frac{\left(v-v_{E}\right)^{2}}{\sigma_{v}^{2}}} \\
& \equiv A v d v e^{-3 \frac{\left(v-v_{E}\right)^{2}}{\sigma_{v}^{2}}},
\end{aligned}
$$

where we have neglected $e^{-3 \frac{\left(v+v_{E}\right)^{2}}{\sigma_{v}^{2}}}$ compared to $e^{-3 \frac{\left(v-v_{E}\right)^{2}}{\sigma_{v}^{2}}}$ and set $A=N 2 \pi \frac{\sigma_{v}^{2}}{3 v_{E}}$. In Eq. (14), we identify $\left|\overrightarrow{v_{E}}\right|=v_{m}$, where $v_{m}$ is the most probable velocity of dark matter. 
[1] N. Aghanim et al. (Planck Collaboration), Planck 2018 results. VI. Cosmological parameters, Astron. Astrophys. 641, A6 (2020).

[2] F. Zwicky, Die Rotverschiebung von extragalaktischen Nebeln, Helv. Phys. Acta 6, 110 (1933).

[3] V. C. Rubin and W. K. Ford, Jr., Rotation of the andromeda nebula from a spectroscopic survey of emission regions, Astrophys. J. 159, 379 (1970).

[4] D. Clowe, M. Bradac, A. H. Gonzalez, M. Markevitch, S. W. Randall, C. Jones, and D. Zaritsky, A direct empirical proof of the existence of dark matter, Astrophys. J. 648, L109 (2006).

[5] E. W. Kolb and M. S. Turner, The Early Universe, Vol. 69 (Addison-Wesley, California, 1990).

[6] S. Tulin and H.-B. Yu, Dark Matter Self-interactions and Small Scale Structure, Phys. Rep. 730, 1 (2018).

[7] J. S. Bullock and M. Boylan-Kolchin, Small-scale challenges to the $\Lambda$ CDM paradigm, Annu. Rev. Astron. Astrophys. 55, 343 (2017).

[8] D. N. Spergel and P. J. Steinhardt, Observational Evidence for Selfinteracting Cold Dark Matter, Phys. Rev. Lett. 84, 3760 (2000).

[9] A. A. de Laix, R. J. Scherrer, and R. K. Schaefer, Constraints of selfinteracting dark matter, Astrophys. J. 452, 495 (1995).

[10] M. R. Buckley and P. J. Fox, Dark matter self-interactions and light force carriers, Phys. Rev. D 81, 083522 (2010).

[11] J. L. Feng, M. Kaplinghat, and H.-B. Yu, Halo Shape and Relic Density Exclusions of Sommerfeld-Enhanced Dark Matter Explanations of Cosmic Ray Excesses, Phys. Rev. Lett. 104, 151301 (2010).

[12] J. L. Feng, M. Kaplinghat, H. Tu, and H.-B. Yu, Hidden charged dark matter, J. Cosmol. Astropart. Phys. 07 (2009) 004.

[13] A. Loeb and N. Weiner, Cores in Dwarf Galaxies from Dark Matter with a Yukawa Potential, Phys. Rev. Lett. 106, 171302 (2011).

[14] J. Zavala, M. Vogelsberger, and M. G. Walker, Constraining Self-Interacting Dark Matter with the Milky Way's dwarf spheroidals, Mon. Not. R. Astron. Soc. 431, L20 (2013).

[15] M. Vogelsberger, J. Zavala, and A. Loeb, Subhaloes in selfinteracting galactic dark matter haloes, Mon. Not. R. Astron. Soc. 423, 3740 (2012).

[16] T. Bringmann, F. Kahlhoefer, K. Schmidt-Hoberg, and P. Walia, Strong Constraints on Self-Interacting Dark Matter with Light Mediators, Phys. Rev. Lett. 118, 141802 (2017).

[17] M. Kaplinghat, S. Tulin, and H.-B. Yu, Dark Matter Halos as Particle Colliders: Unified Solution to Small-Scale Structure Puzzles from Dwarfs to Clusters, Phys. Rev. Lett. 116, 041302 (2016).

[18] L. G. van den Aarssen, T. Bringmann, and C. Pfrommer, Is Dark Matter with Long-Range Interactions a Solution to All Small-Scale Problems of $\Lambda$ CDM Cosmology?, Phys. Rev. Lett. 109, 231301 (2012).

[19] S. Tulin, H.-B. Yu, and K. M. Zurek, Beyond collisionless dark matter: Particle physics dynamics for dark matter halo structure, Phys. Rev. D 87, 115007 (2013).

[20] M. Kaplinghat, S. Tulin, and H.-B. Yu, Direct detection portals for self-interacting dark matter, Phys. Rev. D 89, 035009 (2014).
[21] E. Del Nobile, M. Kaplinghat, and H.-B. Yu, Direct detection signatures of self-interacting dark matter with a light mediator, J. Cosmol. Astropart. Phys. 10 (2015) 055.

[22] C. Kouvaris, I. M. Shoemaker, and K. Tuominen, Selfinteracting dark matter through the Higgs portal, Phys. Rev. D 91, 043519 (2015).

[23] N. Bernal, X. Chu, C. Garcia-Cely, T. Hambye, and B. Zaldivar, Production regimes for self-interacting dark matter, J. Cosmol. Astropart. Phys. 03 (2016) 018.

[24] K. Kainulainen, K. Tuominen, and V. Vaskonen, Selfinteracting dark matter and cosmology of a light scalar mediator, Phys. Rev. D 93, 015016 (2016).

[25] T. Hambye and L. Vanderheyden, Minimal self-interacting dark matter models with light mediator, J. Cosmol. Astropart. Phys. 05 (2020) 001.

[26] M. Cirelli, P. Panci, K. Petraki, F. Sala, and M. Taoso, Dark Matter's secret liaisons: Phenomenology of a dark U(1) sector with bound states, J. Cosmol. Astropart. Phys. 05 (2017) 036.

[27] F. Kahlhoefer, K. Schmidt-Hoberg, and S. Wild, Dark matter self-interactions from a general spin-0 mediator, J. Cosmol. Astropart. Phys. 08 (2017) 003.

[28] E. Aprile et al. (XENON Collaboration), Observation of excess electronic recoil events in XENON1T, Phys. Rev. D 102, 072004 (2020).

[29] F. Takahashi, M. Yamada, and W. Yin, XENON1T Excess from Anomaly-Free Axionlike Dark Matter and Its Implications for Stellar Cooling Anomaly, Phys. Rev. Lett. 125, 161801 (2020).

[30] G. Alonso-Álvarez, F. Ertas, J. Jaeckel, F. Kahlhoefer, and L. J. Thormaehlen, Hidden photon dark matter in the light of XENON1T and stellar cooling, J. Cosmol. Astropart. Phys. 11 (2020) 029.

[31] K. Kannike, M. Raidal, H. Veermäe, A. Strumia, and D. Teresi, Dark matter and the XENON1T electron recoil excess, Phys. Rev. D 102, 095002 (2020).

[32] B. Fornal, P. Sandick, J. Shu, M. Su, and Y. Zhao, Boosted Dark Matter Interpretation of the XENON1T Excess, Phys. Rev. Lett. 125, 161804 (2020).

[33] M. Du, J. Liang, Z. Liu, V. Q. Tran, and Y. Xue, On-shell mediator dark matter models and the XENON1T anomaly, Chin. Phys. C 45, 013114 (2021).

[34] L. Su, W. Wang, L. Wu, J. M. Yang, and B. Zhu, Atmospheric dark matter from inelastic cosmic ray collision in XENON1T, Phys. Rev. D 102, 115028 (2020).

[35] K. Harigaya, Y. Nakai, and M. Suzuki, Inelastic dark matter electron scattering and the XENON1T excess, Phys. Lett. B 809, 135729 (2020).

[36] D. Borah, S. Mahapatra, D. Nanda, and N. Sahu, Inelastic fermion dark matter origin of XENON1T excess with muon $(g-2)$ and light neutrino mass, Phys. Lett. B 811, 135933 (2020).

[37] D. Choudhury, S. Maharana, D. Sachdeva, and V. Sahdev, Dark matter, muon anomalous magnetic moment and the XENON1T excess, Phys. Rev. D 103, 015006 (2021).

[38] J. Bramante and N. Song, Electric But Not Eclectic: Thermal Relic Dark Matter for the XENON1T Excess, Phys. Rev. Lett. 125, 161805 (2020).

[39] N. F. Bell, J. B. Dent, B. Dutta, S. Ghosh, J. Kumar, and J.L. Newstead, Explaining the XENON1T excess with 
Luminous Dark Matter, Phys. Rev. Lett. 125, 161803 (2020).

[40] D. Borah, S. Mahapatra, and N. Sahu, Connecting low scale seesaw for neutrino mass and inelastic sub-GeV dark matter with Abelian gauge symmetry, arXiv:2009.06294.

[41] A. Aboubrahim, M. Klasen, and P. Nath, XENON-1T excess as a possible signal of a sub-GeV hidden sector dark matter, J. High Energy Phys. 02 (2021) 229.

[42] H. M. Lee, Exothermic dark matter for XENON1T excess, J. High Energy Phys. 01 (2021) 019.

[43] S. Baek, J. Kim, and P. Ko, XENON1T excess in local $Z_{2}$ DM models with light dark sector, Phys. Lett. B 810, 135848 (2020).

[44] S. Shakeri, F. Hajkarim, and S.-S. Xue, Shedding new light on sterile neutrinos from xenon1t experiment, J. High Energy Phys. 10 (2020) 194 .

[45] A. Bally, S. Jana, and A. Trautner, Neutrino SelfInteractions and XENON1T Electron Recoil Excess, Phys. Rev. Lett. 125, 161802 (2020).

[46] L. Delle Rose, G. Hütsi, C. Marzo, and L. Marzola, Impact of loop-induced processes on the boosted dark matter interpretation of the XENON1T excess, J. Cosmol. Astropart. Phys. 02 (2021) 031.

[47] Y. Ema, F. Sala, and R. Sato, Dark matter models for the $511 \mathrm{keV}$ galactic line predict keV electron recoils on Earth, Eur. Phys. J. C 81, 129 (2021).

[48] M. Baryakhtar, A. Berlin, H. Liu, and N. Weiner, Electromagnetic signals of inelastic dark matter scattering, arXiv: 2006.13918.

[49] W. Chao, Y. Gao, and M. J. Jin, Pseudo-dirac dark matter in XENON1T, arXiv:2006.16145.

[50] H. An and D. Yang, Direct detection of freeze-in inelastic dark matter, arXiv:2006.15672.

[51] H.-J. He, Y.-C. Wang, and J. Zheng, EFT approach of inelastic dark matter for xenon electron recoil detection, J. Cosmol. Astropart. Phys. 01 (2021) 042.

[52] W.-Y. Keung, D. Marfatia, and P.-Y. Tseng, Stellar cooling, inelastic dark matter, and XENON, arXiv:2009.04444.

[53] H.-J. He, Y.-C. Wang, and J. Zheng, GeV scale inelastic dark matter with dark photon mediator via direct detection and cosmological/laboratory constraints, arXiv:2012.05891.

[54] S.-M. Choi, H. M. Lee, and B. Zhu, Exothermic dark mesons in light of electron recoil excess at XENON1T, arXiv:2012.03713.

[55] D. Tucker-Smith and N. Weiner, Inelastic dark matter, Phys. Rev. D 64, 043502 (2001).

[56] Y. Cui, D. E. Morrissey, D. Poland, and L. Randall, Candidates for inelastic dark matter, J. High Energy Phys. 05 (2009) 076.

[57] R. Adhikari, J. Erler, and E. Ma, Seesaw neutrino mass and new U(1) gauge symmetry, Phys. Lett. B 672, 136 (2009).

[58] D. Borah and R. Adhikari, Abelian gauge extension of Standard Model: Dark matter and radiative neutrino mass, Phys. Rev. D 85, 095002 (2012).

[59] R. Adhikari, D. Borah, and E. Ma, New U(1) gauge model of radiative lepton masses with sterile neutrino and dark matter, Phys. Lett. B 755, 414 (2016).

[60] S. Patra, S. Rao, N. Sahoo, and N. Sahu, Gauged $U(1)_{L_{\mu}-L_{\tau}}$ model in light of muon $g-2$ anomaly, neutrino mass and dark matter phenomenology, Nucl. Phys. B917, 317 (2017).
[61] D. Nanda and D. Borah, Common origin of neutrino mass and dark matter from anomaly cancellation requirements of a $U(1)_{B-L}$ model, Phys. Rev. D 96, 115014 (2017).

[62] B. Barman, D. Borah, P. Ghosh, and A. K. Saha, Flavoured gauge extension of singlet-doublet fermionic dark matter: Neutrino mass, high scale validity and collider signatures, J. High Energy Phys. 10 (2019) 275.

[63] A. Biswas, D. Borah, and D. Nanda, Type III seesaw for neutrino masses in $U(1)_{B-L}$ model with multi-component dark matter, J. High Energy Phys. 12 (2019) 109.

[64] D. Nanda and D. Borah, Connecting light dirac neutrinos to a multi-component dark matter scenario in gauged $B-L$ model, Eur. Phys. J. C 80, 557 (2020).

[65] M. Dutta, S. Bhattacharya, P. Ghosh, and N. Sahu, Singletdoublet majorana dark matter and neutrino mass in a minimal type-I seesaw scenario, J. Cosmol. Astropart. Phys. 03 (2021) 008.

[66] S. Mahapatra, N. Narendra, and N. Sahu, Verifiable type-II seesaw and dark matter in a gauged $U(1)_{B-L}$ model, arXiv: 2002.07000 .

[67] N. Okada and O. Seto, Inelastic extra $U(1)$ charged scalar dark matter, Phys. Rev. D 101, 023522 (2020).

[68] K. Schutz and T. R. Slatyer, Self-scattering for dark matter with an excited state, J. Cosmol. Astropart. Phys. 01 (2015) 021.

[69] M. Blennow, S. Clementz, and J. Herrero-Garcia, Selfinteracting inelastic dark matter: A viable solution to the small scale structure problems, J. Cosmol. Astropart. Phys. 03 (2017) 048.

[70] Y. Zhang, Self-interacting dark matter without direct detection constraints, Phys. Dark Universe 15, 82 (2017).

[71] N. Arkani-Hamed, D. P. Finkbeiner, T. R. Slatyer, and N. Weiner, A theory of Dark Matter, Phys. Rev. D 79, 015014 (2009).

[72] A. Kamada, H. J. Kim, and T. Kuwahara, Maximally selfinteracting dark matter: Models and predictions, J. High Energy Phys. 12 (2020) 202.

[73] G. Alvarez and H.-B. Yu, Astrophysical probes of inelastic dark matter with a light mediator, Phys. Rev. D 101, 043002 (2020).

[74] A. Semenov, LanHEP-A package for automatic generation of Feynman rules from the Lagrangian. Version 3.2, Comput. Phys. Commun. 201, 167 (2016).

[75] A. Belyaev, N. D. Christensen, and A. Pukhov, CalcHEP 3.4 for collider physics within and beyond the Standard Model, Comput. Phys. Commun. 184, 1729 (2013).

[76] Wolfram Research, Inc., Mathematica, Version 12.2, December 2020.

[77] L. J. Hall, K. Jedamzik, J. March-Russell, and S. M. West, Freeze-in production of FIMP dark matter, J. High Energy Phys. 03 (2010) 080.

[78] D. Borah, P. S. B. Dev, and A. Kumar, TeV scale leptogenesis, inflaton dark matter and neutrino mass in a scotogenic model, Phys. Rev. D 99, 055012 (2019).

[79] J. L. Feng, A. Rajaraman, and F. Takayama, SuperWIMP dark matter signals from the early universe, Phys. Rev. D 68, 063504 (2003).

[80] T. R. Slatyer, The Sommerfeld enhancement for dark matter with an excited state, J. Cosmol. Astropart. Phys. 02 (2010) 028. 
[81] B. M. Roberts and V. V. Flambaum, Electron-interacting dark matter: Implications from DAMA/LIBRA-phase 2 and prospects for liquid xenon detectors and NaI detectors, Phys. Rev. D 100, 063017 (2019).

[82] B. M. Roberts, V. A. Dzuba, V. V. Flambaum, M. Pospelov, and Y. V. Stadnik, Dark matter scattering on electrons: Accurate calculations of atomic excitations and implications for the DAMA signal, Phys. Rev. D 93, 115037 (2016).

[83] T. Piffl et al., The RAVE survey: The Galactic escape speed and the mass of the Milky Way, Astron. Astrophys. 562, A91 (2014).

[84] A. Abdelhameed et al. (CRESST Collaboration), First results from the CRESST-III low-mass dark matter program, Phys. Rev. D 100, 102002 (2019).

[85] D. S. Akerib et al. (LUX Collaboration), Results of a Search for Sub-GeV Dark Matter Using 2013 LUX Data, Phys. Rev. Lett. 122, 131301 (2019).
[86] Q. Arnaud et al. (NEWS-G Collaboration), First results from the NEWS-G direct dark matter search experiment at the LSM, Astropart. Phys. 97, 54 (2018).

[87] E. Armengaud et al. (EDELWEISS Collaboration), Searching for low-mass dark matter particles with a massive $\mathrm{Ge}$ bolometer operated above-ground, Phys. Rev. D 99, 082003 (2019).

[88] J. Lees et al. (BABAR Collaboration), Search for a Dark Photon in $e^{+} e^{-}$Collisions at BABAR, Phys. Rev. Lett. 113, 201801 (2014).

[89] M. Ibe, S. Kobayashi, Y. Nakayama, and S. Shirai, Cosmological constraint on dark photon from $N_{\text {eff }}$, J. High Energy Phys. 04 (2020) 009.

[90] P. Gondolo and G. Gelmini, Cosmic abundances of stable particles: Improved analysis, Nucl. Phys. B360, 145 (1991). 